\title{
Ultrasound stimulation improves inflammatory resolution, neuroprotection, and functional recovery after spinal cord injury
}

\section{Yu-ri Hong}

Daegu-Gyeongbuk Medical Innovation Foundation

\section{Eun-hee Lee}

Daegu-Gyeongbuk Medical Innovation Foundation

Ki-su Park

Kyungpook National University School of Medicine

\section{Mun Han}

Daegu-Gyeongbuk Medical Innovation Foundation

\section{Kyoung-Tae Kim}

Kyungpook National University, Kyungpook National University Hospital

Juyoung Park ( $\nabla$ jyp@dgmif.re.kr)

Daegu-Gyeongbuk Medical Innovation Foundation

\section{Research Article}

Keywords: Spinal cord injury, ultrasound stimulation, SCIU5, SCIU40

Posted Date: April 15th, 2021

DOI: https://doi.org/10.21203/rs.3.rs-413315/v1

License: (c) (1) This work is licensed under a Creative Commons Attribution 4.0 International License.

Read Full License

Version of Record: A version of this preprint was published at Scientific Reports on March 7th, 2022. See the published version at https://doi.org/10.1038/s41598-022-07114-6. 
4 Yu-ri Hong ${ }^{1, \dagger}$, Eun-hee Lee ${ }^{1, \dagger}$, Ki-su Park ${ }^{2}$, Mun Han ${ }^{1}$, Kyoung-Tae Kim ${ }^{3}$ *, Juyoung Park ${ }^{1, *}$

5

$6 \quad{ }^{1}$ Medical Device Development Center, Daegu-Gyeongbuk Medical Innovation Foundation, Daegu, Republic of $7 \quad$ Korea

$8 \quad{ }^{2}$ Department of Neurosurgery, Kyungpook National University School of Medicine, Daegu, Korea

$9{ }^{3}$ Department of Neurosurgery, School of Medicine, Kyungpook National University, Kyungpook National 10 University Hospital, Daegu, Korea

12 'The authors contributed equally to this work.

$14 *$ Corresponding authors:

15 Kyoung-Tae Kim, M.D., Ph.D., Department of Neurosurgery, School of Medicine, Kyungpook National 16 University, Kyungpook National University Hospital, 130 Dongdeok-ro, Jung-gu, Daegu 41944, Korea, E17 mail: nskimkt7@gmail.com Juyoung Park. Daegu-Gyeongbuk Medical Innovation Foundation, Medical Device Development Center, Daegu, 41061, South Korea; E-mail: opedoors@gmail.com 


\section{Abstract}

23 Spinal cord injury (SCI) is associated with limited functional recovery. Despite advances in neuroscience, realistic 24 therapeutic treatments for SCI remain unavailable. In this study, the effects of non-invasive ultrasound (US) 25 treatment on behavior and inflammatory responses were evaluated in a rat model of SCI. Adult female Sprague26 Dawley rats were subjected to spinal cord contusion injury. Two different US parameters (SCIU5: 5\% and SCIU40: $2740 \%$ duty cycle) were applied, and their effects on behavioral recovery after SCI were quantified. Tissue and neuronal responses were detected. Immunofluorescence was used to detect inflammatory markers. In the rat model of SCI, motor function was more effectively restored, and the lesion cavity area was smaller in the SCIU5 group. Furthermore, the SCIU5 protocol elicited an anti-inflammatory response at the injury site by reducing degenerative FJC-labeled neurons, macrophage/microglia activation, and infiltration. Thus, the lesion area decreased, and tissue density increased. Meanwhile, the SCIU40 protocol did not improve motor function or induce an anti-inflammatory response at the injury site. The SCIU5 protocol effectively accelerated the rate of improved exercise performance in the rat model while reducing inflammation. Accordingly, appropriate US stimulation may represent a promising treatment modality for SCI with beneficial anti-inflammatory effects. 
Introduction

Spinal cord injury (SCI) is associated with the severe and permanent impairment of motor and sensory functions. Functional deficits are induced by an initial mechanical injury and subsequent secondary injury lasting weeks or months ${ }^{1-3}$. Secondary injuries occur via a cascade of biochemical and pathological changes that exacerbate tissue damage, including inflammatory cell infiltration, oxidative stress, ischemia, inflammatory cytokine release, and initiation of apoptotic signaling cascades ${ }^{4,5}$. Therefore, therapeutic approaches should focus on early phase treatment to prevent damage induced by secondary pathophysiological processes. Several SCI treatment approaches have been evaluated, including surgical interventions ${ }^{6}$, pharmacological approaches ${ }^{7-9}$, stem cell treatment ${ }^{10,11}$, biomaterials ${ }^{12,13}$, and functional electrical stimulation ${ }^{14,15}$. However, studies examining the efficacy of these therapeutic strategies remain limited, and their application is highly debated owing to potential side effects and safety concerns ${ }^{16-18}$. Therefore, the development of effective, simple, and stable therapies for SCI is urgently needed.

The therapeutic application of ultrasound (US) has progressed substantially since the $1950 \mathrm{~s}{ }^{19-21}$. US energy deposited in tissues can induce biological effects via radiation and mechanical stress. US, alone or combined with microbubbles, is widely used for the treatment of various malignant human cancers ${ }^{22-24}$, drug delivery ${ }^{25}$, and curative therapies ${ }^{26}$. Moreover, several recent clinical trials and experimental studies have reported that US can elicit an anti-inflammatory response, promote tissue repair, and reduce pain ${ }^{27-30}$. US stimulation reduces inflammatory mediators, such as cyclooxygenase (COX)-1/2, inducible nitric oxide synthase (iNOS), tumor necrosis factor (TNF)- $\alpha$, and interleukin (IL)-1 $\beta$, as well as immune cell infiltration, in numerous inflammatory diseases, including peripheral nerve injury ${ }^{31-33}$. In particular, Zachs et al. demonstrated that daily US stimulation targeting the spleen could induce an anti-inflammatory response via immunomodulation of both $\mathrm{T}$ and $\mathrm{B}$ cells, conferring protective and therapeutic effects in inflammatory arthritis ${ }^{34}$. The non-invasive modulation of specific neuronal signaling pathways within the spleen circuit demonstrates the potential therapeutic benefits of US for inflammatory diseases. Furthermore, preclinical studies have reported transcranial US stimulation results in functional recovery and neuroprotective effects in neurodegenerative diseases via induction of brain-derived neurotrophic factor (BDNF) ${ }^{35,36}$. These results raise the possibility that US stimulation could block inflammatory reactions and neuronal damage while promoting neuronal regeneration.

In the current study, we investigated whether US stimulation has a therapeutic effect in an SCI rat model. We demonstrated that US stimulation improves locomotor behavior, reduces tissue/neuronal damage, and 
66 inflammatory responses by infiltrating immune cells. The observed anti-inflammatory effects support the potential 67 clinical application of US to develop efficient and safe SCI treatments.

\section{Results}

70

\section{Contusion force classification for optimal SCI induction}

1 Various degrees of force, ranging from 140 to $200 \mathrm{Kdyn}$, were evaluated (Figure 1 and Table 1) to obtain the optimal level of bruising in the SCI model for short- and long-term experiments (Table 1 and Figure S1). In rats administered 150 and $200 \mathrm{Kdyn}$, the average Basso, Beattie, and Bresnahan (BBB) score was $0.3 \pm 0.5(P=0.008)$ on day 5 after the injury, while that for 140 Kdyn was $3.4 \pm 2.8$. After 7 days, the mean BBB scores for 150 and 200 Kdyn were $0.8 \pm 0.8(P<0.0001)$ and $1.3 \pm 1.0(P<0.0001)$, respectively, which differed significantly from that for $140 \mathrm{Kdyn}$ (i.e., $8.5 \pm 2.2$ ). Initial studies revealed that $140 \mathrm{Kdyn}$ was an intermediate level of exposure at the T10 level of the spinal cord and was used for subsequent analyses. 
A

Stereotactic frame basedultrasound stimulation system

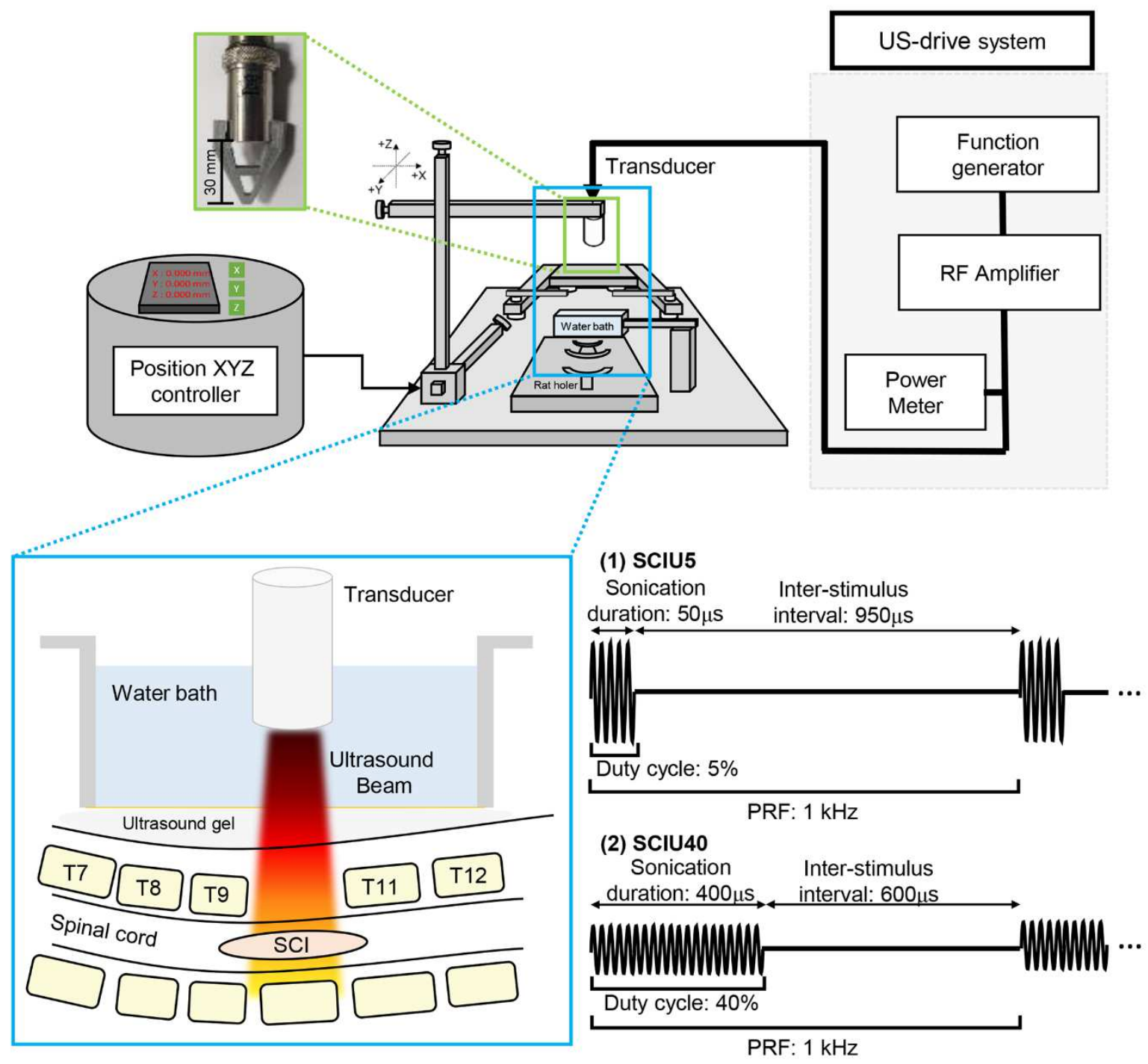

B

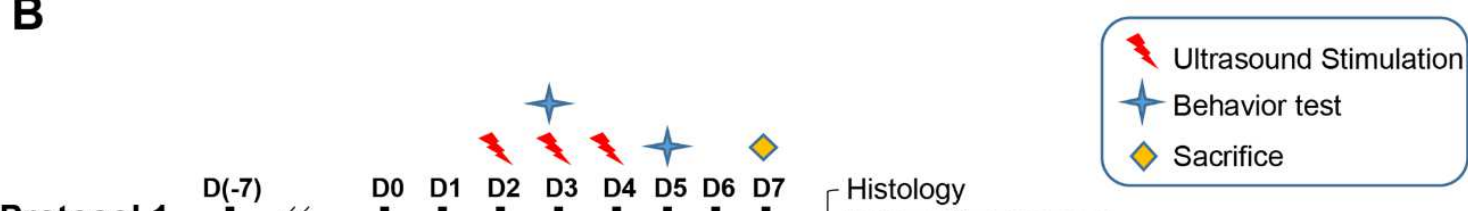

Protocol 1 D(-7)

Pre-train SCI model

rat

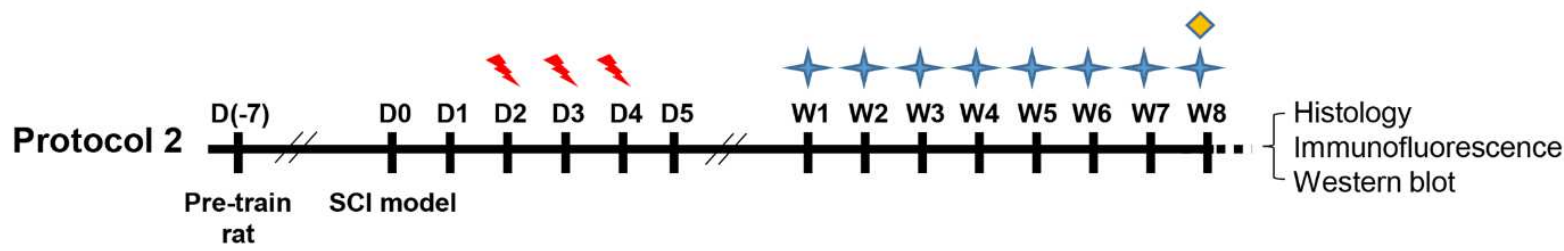




\section{Improvement of locomotor function during the acute SCI phase}

We first investigated US treatment effects in the acute SCI phase (Table 2 and Figure S2). Following US stimulation for 2 to 4 days, functional motor recovery was assessed by BBB scores and the ladder rung test in both the SCIU5 and SCIU40 groups. Although scores were very low 3 days after SCI, slight differences were observed among the three experimental groups even at this early time point. The BBB score for the SCIU5 treatment group was $3.5 \pm 3.2$ compared to $1.9 \pm 2.4(P=0.1)$ for the sham control and $1.5 \pm 1.5(P=0.04)$ for the SCIU40 treatment group (Figure 2a). At 5 and 7 days after spinal contusion, the BBB scores for the SCIU5treatment group were $6.8 \pm 2.5$ and $13.3 \pm 2.5$, respectively, which differed significantly from those in the sham control group $(4.3 \pm 1.5(P=0.007)$ and $9.9 \pm 2.6(P=0.0002)$, respectively). Meanwhile, on days 5 and 7 postinjury, the SCIU40 treatment group had BBB scores of $3.8 \pm 1.8(P=0.8)$ and $8.7 \pm 2.8(P=0.4)$, similar to those in the sham control group. Similarly, the average number of hindlimb errors in the SCIU5 treatment group improved significantly to $20.3 \pm 5.3$ mistakes compared to the sham control $(23.5 \pm 5.7$ mistakes, $P=0.04)$ and SCIU40 treatment group ( $25.9 \pm 6.3$ mistakes, $P=0.0003)$, at 7 days (Figure $2 \mathbf{b})$. These results suggest that US treatment under the SCIU5 condition could improve hindlimb motor function recovery after SCI. In the SCIU40 group, hind leg motor function recovered similar to the sham control group.

\section{Reduction of tissue and neuronal cell damage in the acute SCI phase}

Lesion cavity volumes were assessed in serial hematoxylin and eosin (H\&E)-stained spinal cord sections in the transverse plane after 7 days. The lesion cavity areas relative to that in the sham control group were $40.9 \pm$ $14.0 \%(P<0.0001)$ in the SCIU5-treated group and $123.6 \pm 16.4 \%(P=0.0005)$ in the SCIU40-treated group (Figure 2c, e). The tissue densities around the lesion were significantly higher in the SCIU5 and SCIU40 groups than in the sham control group (Figure 2d, f). These results were confirmed by in vivo T2W MR images to identify structural changes at the spinal cord lesion site corresponding to histological features (Figure S3). At the lesion center, signal intensity at the lesion center, representing atrophy caused by hemorrhage, edema, and fibroglial scarring, was lower in the SCIU5-treated group than in the sham control SCIU40-treated group 14 days after SCI. We used Fluoro-Jade C (FJC) staining to label degenerating neurons in the damaged cord tissue to evaluate the neuroprotective effects. We detected $98.4 \pm 25.8$ FJC-positive cells in the SCIU5-treated group, which was significantly fewer than that of the sham control $(183.0 \pm 77.9 ; P=0.001)$ and SCIU40-treated $(154.4 \pm 40.5 ; P$ 
$107=0.04$ ) groups (Figure $2 \mathbf{g}, \mathbf{h}$ ). Furthermore, the FJC signal intensity was $0.7 \pm 0.4$ in the SCIU5- treated group,

108 which was significantly lower than those in the sham control $(1.0 \pm 0.6, P=0.0007)$ and SCIU40-treated group $109(1.3 \pm 0.6, P<0.0001 ;$ Figure 2i). These results suggest that SCIU40 treatment tends to increase nerve damage 110 and that SCIU5 treatment has an inhibitory effect on tissue and nerve damage in acute SCI. 
A

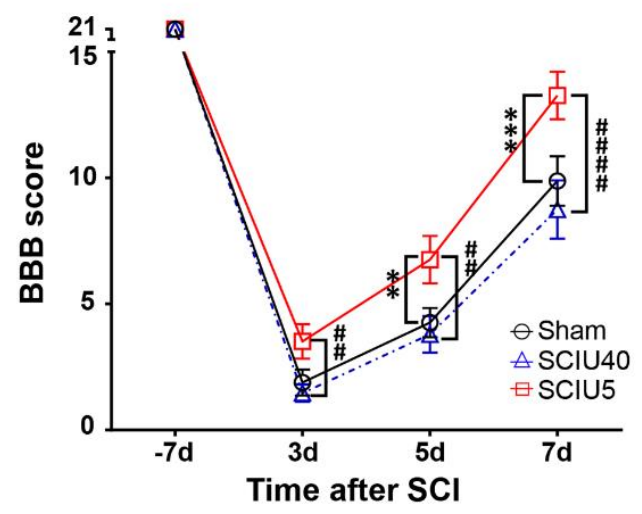

C

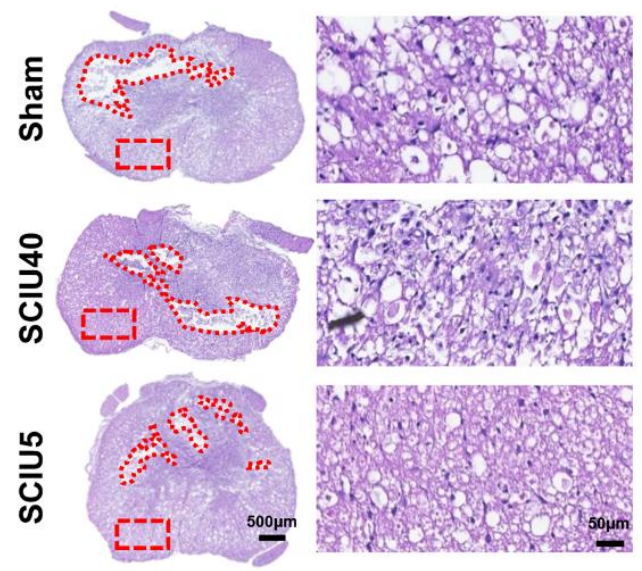

E

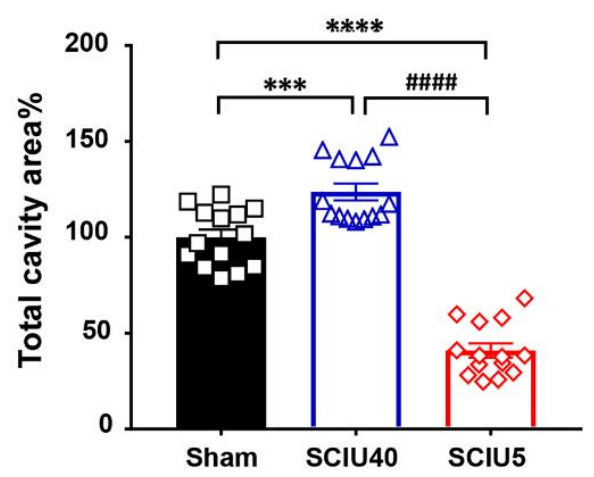

F

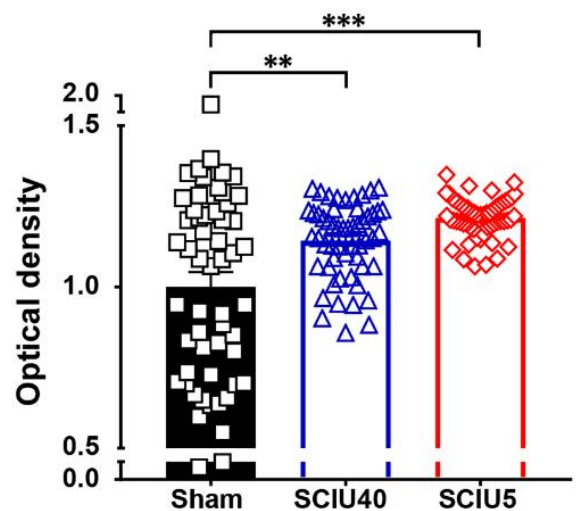

B

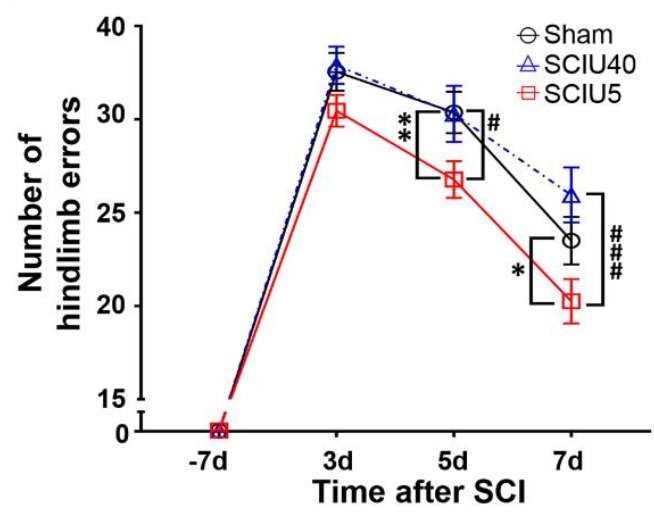

G
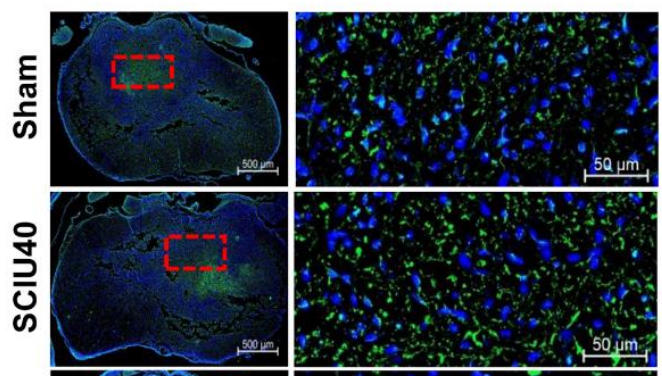

응

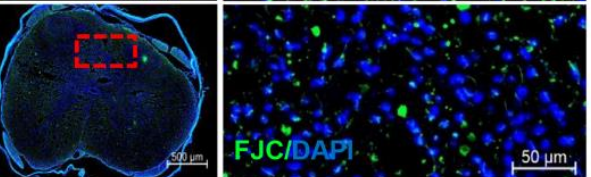

H

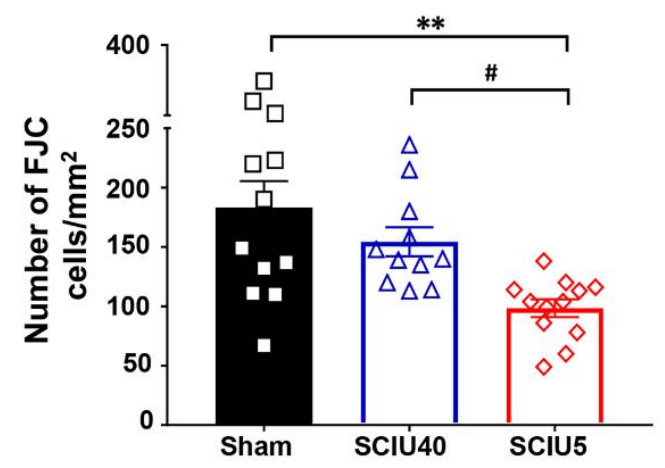

I

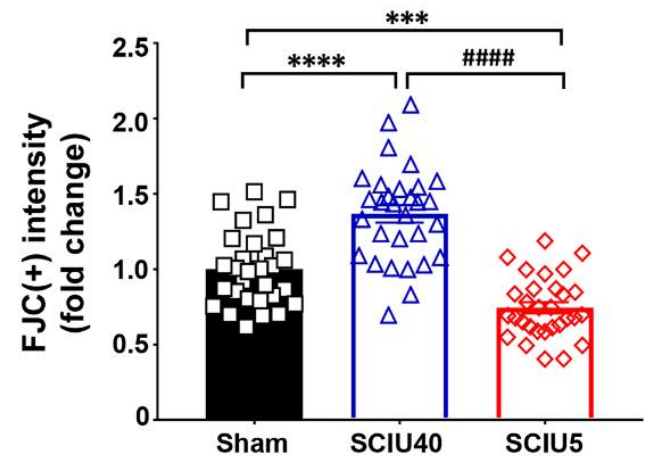


114 To investigate the effect of US treatment on the inflammatory response after SCI, immunofluorescent 115 detection of ED-1 (macrophage/microglial cell marker), iNOS, and TNF- $\alpha$ was performed to determine the level 116 of macrophage/microglial cell activation (Figure 3 and Figure S4). The relative fluorescence intensity of ED-1117 positive macrophages/microglia was low in the SCIU5-treated group compared with the sham control group 7 days after spinal contusion $(0.65 \pm 0.3$-fold change, $P=0.01)$. In contrast, the SCIU40-treated group showed an 119 increase in relative intensity compared with that in the sham control group $(1.12 \pm 0.1$-fold; $P=0.5$; Figure $3 \mathbf{g})$. Furthermore, levels of iNOS and TNF- $\alpha$, indicators of the macrophage-induced inflammatory response, were substantially reduced in the SCIU5-treated group, with relative changes of $0.7 \pm 0.2$ - fold $(P=0.1)$ and $0.3 \pm 0.3$ fold $(P<0.0001)$ compared to the sham control group (Figure 3h, i). Meanwhile, the SCIU40 treatment group showed no differences in iNOS and TNF- $\alpha$ levels compared to those in the sham control group (fold changes: 1.1 $\pm 0.5(P=0.6)$ and $1.0 \pm 0.3(P<0.9)$, respectively). These findings were confirmed by western blot analysis of pro-inflammatory cytokines in whole damaged tissues (Figure 3j). In the SCIU5- treated group, the expression levels of ED-1, iNOS, and TNF- $\alpha$ were dramatically lower than those in the sham control and SCIU40-treated groups. Similarly, expression levels were lower in the SCIU40-treated group than in the sham control group. These results indicate that SCIU5 treatment has an inhibitory effect on the production of pro-inflammatory factors caused by the inflammatory response and immune cell infiltration in the acute injury stage. 

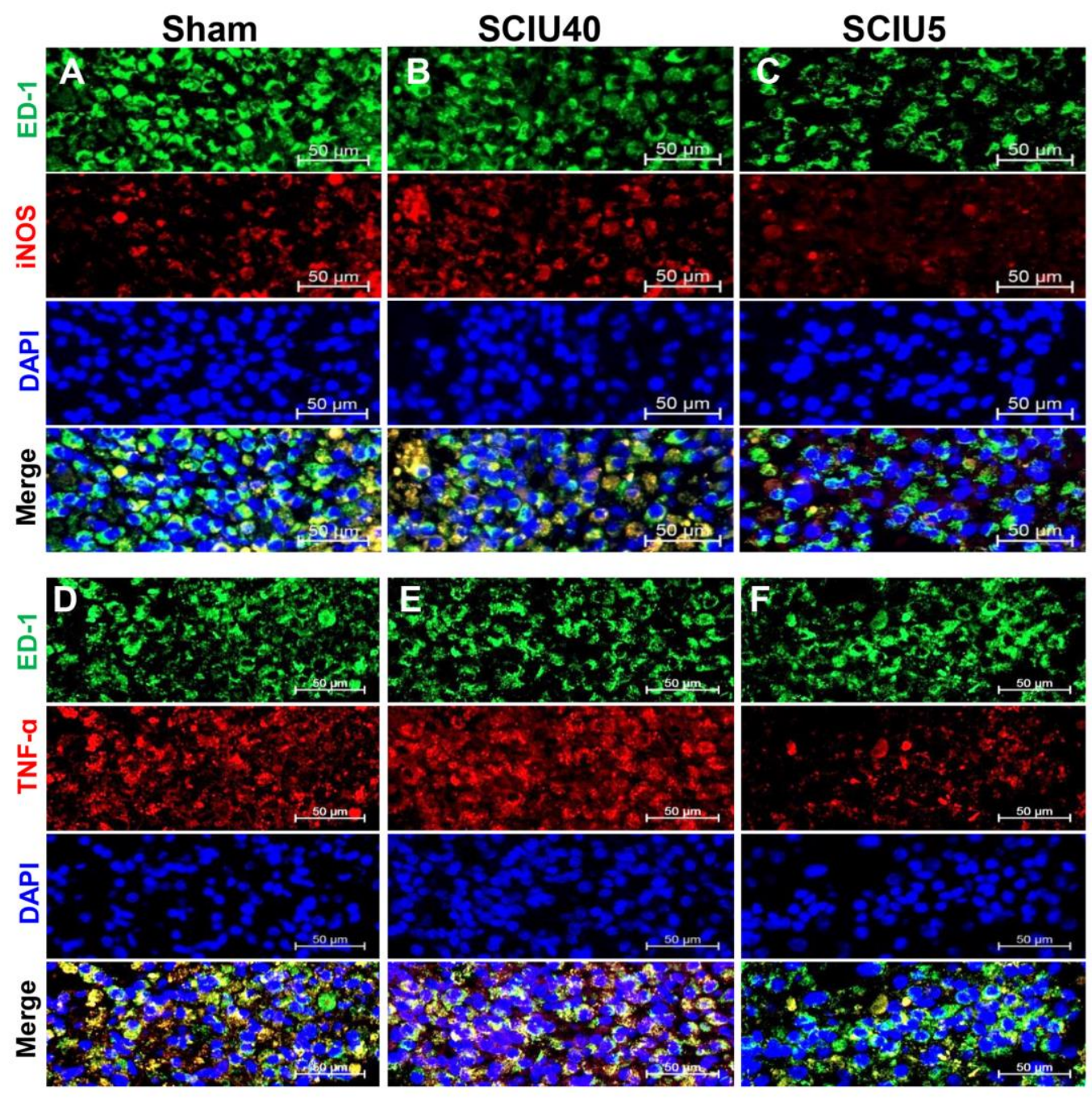

G

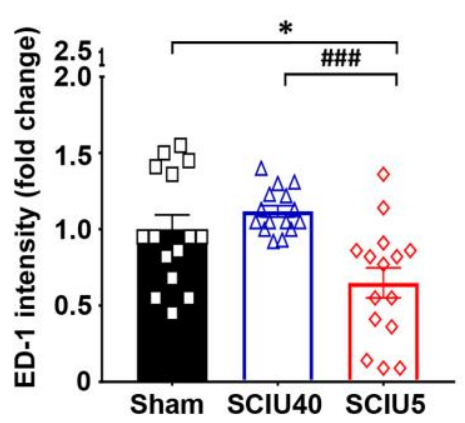

J

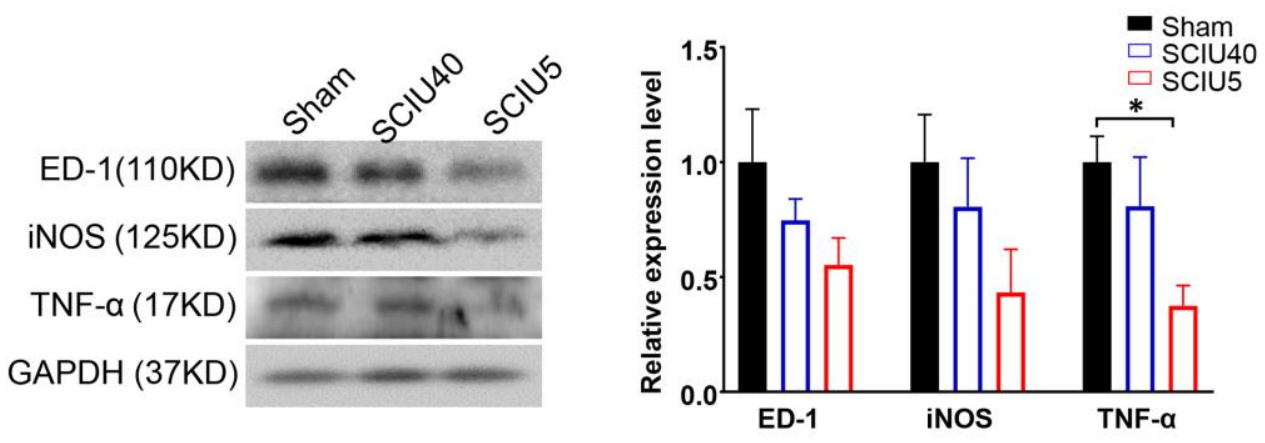

H
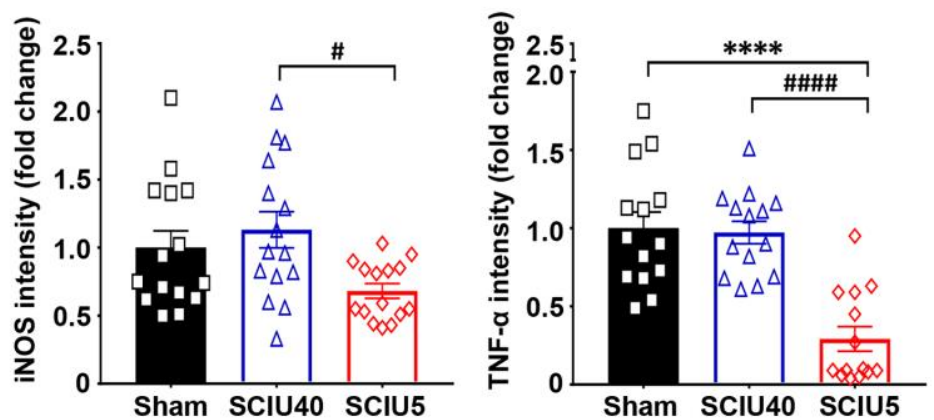


\section{Locomotor recovery in the chronic SCI phase}

Given the therapeutic effects of US treatment in the acute SCI phase, we tested whether this approach could also lead to locomotor function recovery in the chronic SCI phase. Functional locomotor tests were performed once per week for 8 weeks. (Figure 1b, Protocol 2). Hind limb locomotor activity showed rapid recovery during the first 3 weeks and continued thereafter at a slower rate, with a BBB score similar to that of normal rats (i.e., 21). During the recovery period, the US-treated groups showed significantly improved motor function compared to the sham control group in the open-field test (Figure 4a). One week later, the BBB scores in the SCIU5-treated group were $13.4 \pm 3.1$, whereas scores in the SCIU40-treated group $(8.9 \pm 3.1, P=0.03)$ and sham control group $(8.0 \pm 3.2, P=0.02)$ were $>5$ points lower. Two weeks after spinal injury, the sham control and SCIU40-treated groups had BBB scores of $16.7 \pm 1.5(P=0.04)$ and $16.1 \pm 2.6(P=0.1)$, respectively, which was higher than that of the SCIU5-treated group $(12.8 \pm 4.4)$. In the horizontal ladder rung walking test, the SCIU5-treated group showed significantly improved recovery of hindlimb motor activity, whereas the SCIU40-treated group showed an error similar to that of the sham control group until 8 weeks after SCI (Figure $4 \mathbf{b}$ ). These results suggest that US treatment can improve hindlimb motor function in the chronic SCI phase.

\section{Reduction of tissue and neuronal cell damage in the chronic SCI phase}

To investigate the positive therapeutic effect on tissue damage 8 weeks after treatment, the lesion cavity and neuronal cell death were assessed histologically. Upon histological evaluation, the lesion cavity areas relative to that in the sham control group were $67.5 \pm 33.5 \%(P=0.05)$ in the SCIU5-treated group and $136.5 \pm 36.0 \%(P=$ $0.03)$ in the SCIU40-treated group. Tissue density was $1.2 \pm 0.3$-fold $(P<0.0001)$ higher in the SCIU5- treated group than in the sham control group. In contrast, in the SCIU40-treated group, the lesion cavity occupancy rate was higher, and the tissue density was lower by $0.8 \pm 0.0$-fold $(P<0.0002)$ than those in the sham control group (Figure 4c-f). We further analyzed FJC-positive neural cell damage. The number of FJC-positive cells in the SCIU5-treated group $(279.1 \pm 177.5)$ was significantly lower than that in the sham control $(511.3 \pm 317.2, P=$ $0.02)$ and SCIU40-treated $(383.9 \pm 163.8, P=0.4)$ groups (Figure $4 \mathbf{g}, \mathbf{h})$. Furthermore, the FJC fluorescence intensities were significantly lower in the SCIU5-treated $(2.6 \pm 2.4, P<0.0001)$ and SCIU40-treated $(3.2 \pm 2.6$, $P=0.4)$ groups than in the sham control $(4.9 \pm 2.4)$. We did not find a significant reduction in the lesion cavity area or neuronal cell death in the SCIU40 treatment group. Nevertheless, these results strongly suggest that SCIU5 therapy can be employed to prevent further damage to spinal nerve dysfunction, even in the chronic stage of SCI. 
A

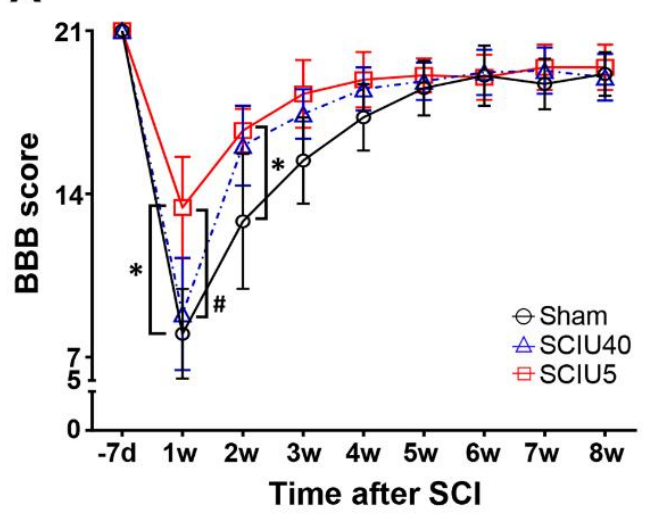

C

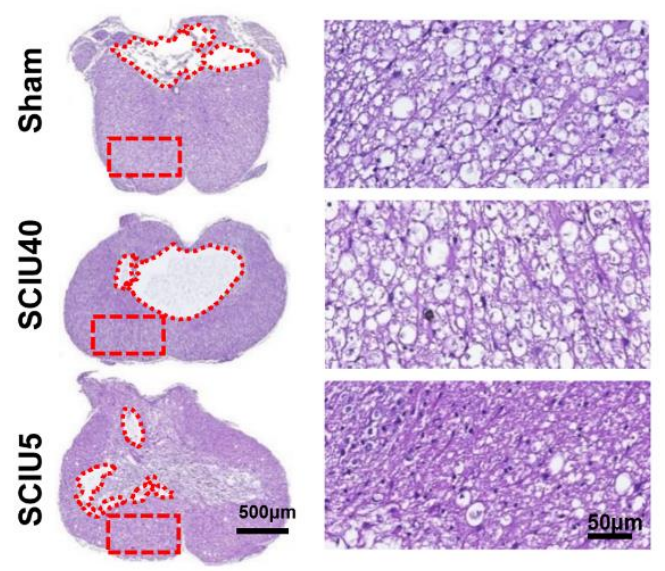

E

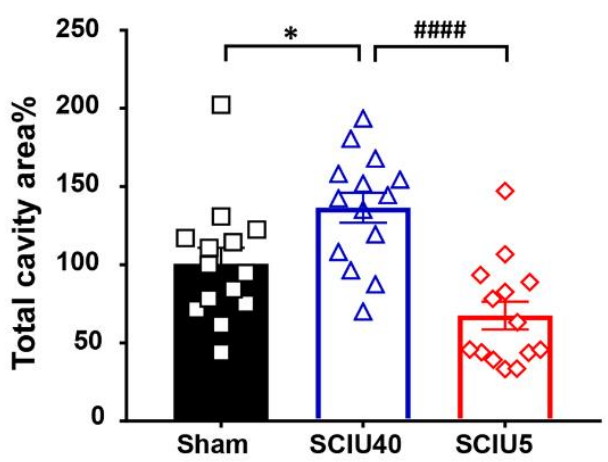

F

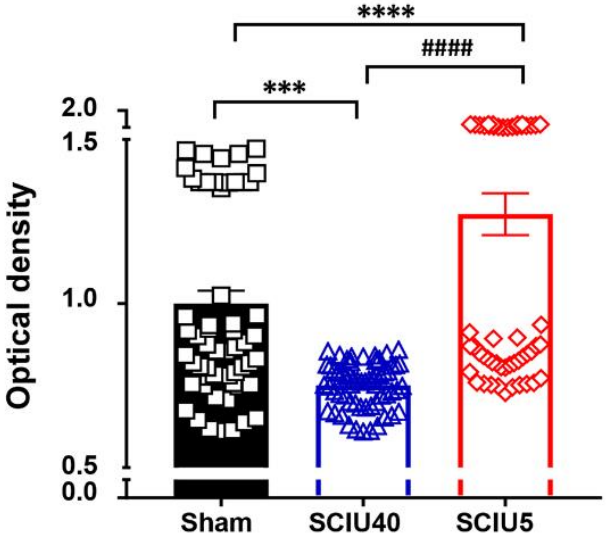

B

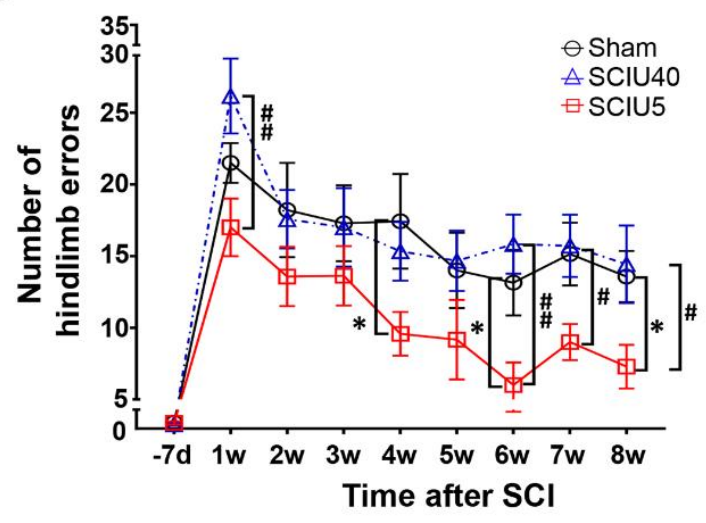

G
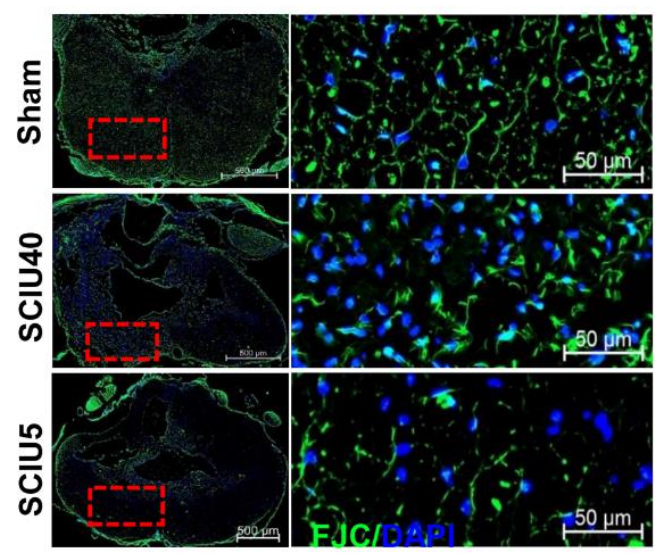

H

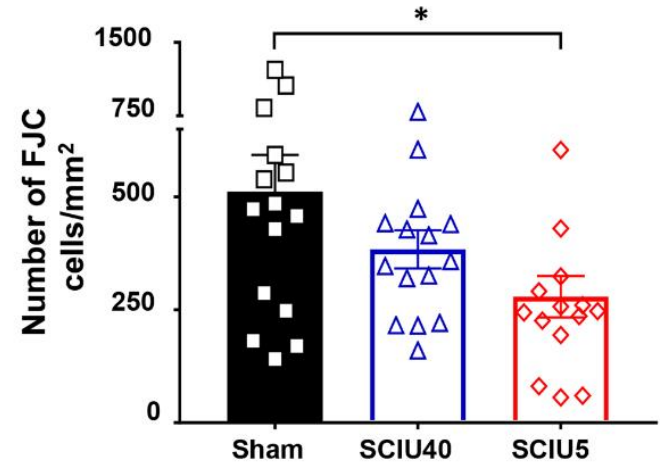

I

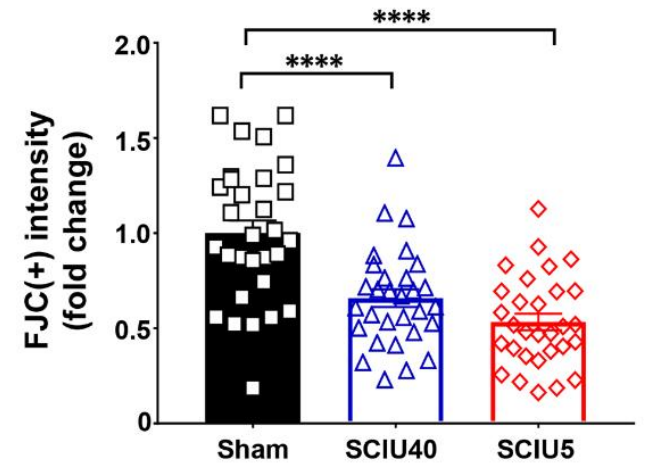




\section{Discussion}

US stimulation can induce several biological effects mediated by radiations and mechanical stress. Recently, preclinical studies have reported that US application results in functional recovery and has neuroprotective effects in neurodegenerative and traumatic diseases via modulation of inflammation and neuroregeneration processes (via BDNF) ${ }^{35,36}$. This study showed that low-intensity US stimulation could induce the recovery of hindlimb dyskinesia in rats with a damaged spinal cord. Two US treatment conditions (SCIU5 and SCIU40) were evaluated to confirm their effectiveness. The SCIU5 treatment group showed a significant improvement in functional motor outcomes, supported by significant improvements in the BBB score and ladder rung test in the acute SCI model (Figure 2a, b). In particular, in the chronic SCI model (Figure 4a, b), the SCIU5 treatment group maintained a significantly higher rate of functional motor improvement in the ladder rung test compared to those in other conditions up to 8 weeks of follow-up. The performance improvement in the ladder rung test might be related to the recovery of the spinal circuit communication between the supraspinal and spinal circuits, which is essential for performance in the ladder rung test ${ }^{37}$.

Similar to the motor function results, histological improvements in the SCIU5 treatment group were confirmed in our study. The lesion cavity size and neuronal cell damage in the SCIU5 treatment group were significantly decreased in the acute SCI model (Figure 2c-i) and the chronic SCI model (Figure 4c-i). Indeed, low-intensity pulsed US therapy has been reported to modulate cytokine production, phagocytosis, and the expression of genes related to tissue repair ${ }^{38,39}$. US treatment can also increase neurotrophic factor protein levels to suppress apoptosis and protect nerves in an animal model of traumatic brain injury ${ }^{40}$. Hence, the observed histopathological improvements could provide evidence of anti-inflammatory effects by inducing neurotrophic factors and tissue repair by US stimulation

In previous studies, edema and neutrophil infiltration in a rodent model of foot edema were significantly reduced by low-intensity ultrasound stimulation ${ }^{41,42}$. Similarly, we observed the development of edema (Figure S3) and increased levels of inflammatory factors (ED-1, iNOS, and TNF- $\alpha$; Figure 3) within the SCI area, all of which was reduced following SCIU5 treatment. Furthermore, the group treated with SCIU5 showed decreased macrophage marker ED-1, indicating that the response to US stimulation was closely related to the regulation of inflammatory cell infiltration. The expression of iNOS is strongly linked to activated macrophages and accelerates tissue damage following SCI ${ }^{43,44}$. In the SCIU5 treatment group, iNOS expression was significantly reduced in the damaged spinal cord, indicating a prominent decrease in neurodegeneration and neuroinflammation. 
Furthermore, SCIU40 caused a slight increase in inflammatory factor expressions (ED-1, iNOS, and TNF- $\alpha$ ).

191

192

193

194

195

196

197

198

199

200

201

202

203

204

205

206

207

208

209

210

211

212

213

214

215

216

217

These results indicate that the inflammatory cytokine response may vary depending on US treatment conditions. Although further detailed studies of the inflammatory response are needed, US therapy is a promising SCI strategy aimed at reducing inflammatory factors.

Recent studies have suggested that ultrasound stimulation parameters, according to the differential duty cycle, affect the activation (at high duty cycles) and suppression (at low duty cycles) of the three cortical neuronal with the same ultrasound intensities ${ }^{45,46}$. We, therefore, hypothesized that SCIU5 (5\% duty cycle mode) and SCIU40 ( $40 \%$ duty cycle mode), with the same intensity $\left(0.8 \mathrm{~W} / \mathrm{cm}^{2}\right)$, would elicit neuronal modulation and inflammatory responses in SCI. However, our observations provide the first evidence that SCIU5, a suppression US condition, suppresses the inflammatory response and enhances the locomotor functions, whereas SCIU40, an activation US condition, did not significantly improve functional outcomes (Figures 2 and 4) or inflammatory responses (Figure 3). Considering that we applied US treatment during the acute phase (2-4 days after SCI), which is associated with a massive inflammatory response ${ }^{47}$, it is reasonable to speculate that the suppressive US parameter induced by low duty cycles might be more effective than activation conditions when treating acute phase SCI.

In future studies, we will seek to establish an appropriate US protocol to effectively reduce inflammation and promote nerve regeneration via a combination of the SCIU5 and SCIU40 protocols. Taken together, US treatment with the SCIU5 protocol could improve motor function while reducing inflammation and tissue damage in a rat model of SCI. These results suggest that US stimulation is a promising non-invasive treatment modality for SCI.

\section{Methods}

\section{Animals}

A total of 105 adult female Sprague-Dawley rats (weighing $200 \pm 40$ g; Orient Bio Inc., Seongnam, Korea) were used for all the experiments (Table 1). The study was approved by the Daegu-Gyeongbuk Medical Innovation Foundation (DGMIF) and the Institutional Animal Care and Use Committee (IACUC, DGMIF-19040101-00). Rats were maintained under specific pathogen-free conditions in individual ventilating systems; The rats were fed a normal pellet diet and water ad libitum. They were housed at a temperature of $22 \pm 1{ }^{\circ} \mathrm{C}$ with a relative humidity of 50\% $\pm 10 \%$, using $12 \mathrm{~h} \mathrm{light/dark} \mathrm{cycles,} \mathrm{illumination} \mathrm{at} \mathrm{150-300} \mathrm{Lux} \mathrm{and} \mathrm{ventilation} \mathrm{10-20} \mathrm{times/hour.}$ 
218 Animals were anesthetized for all the experimental procedures and were constantly monitored throughout the 219 course of the experiment. Animal handling and all the procedures were performed in accordance with the ethical 220 guidelines for animal studies; the experiments were carried out in compliance with the appropriate Animal

221 Research: Reporting In Vivo Experiments (ARRIVE) guidelines.

\section{Establishment of the SCI model}

Rats were anesthetized with a combination of Zoletil $25 \mathrm{mg} / \mathrm{kg}$ (Virbac Laboratories, Carros, France) and Rumpun (4.6 mg/kg; Bayer, Leverkusen, Germany). The fur was then shaved from the backs of rats using a depilatory cream. Dorsal laminectomy was performed at the T10 level. Following laminectomy, each animal received kanamycin sulfate $(0.05 \mathrm{mg} / \mathrm{kg}$; Yuhan, Seoul, Korea). SCI was induced using an Infinite Horizon (IH) SCI device (Precision Systems \& Instrumentation, Lexington, KY, USA) ${ }^{48}$. To evaluate the optimal contusion level for the long and short-term experiments, various degrees of force were evaluated from 140 to $200 \mathrm{Kdyn}$. The detailed parameters for the IH impactor are summarized in Table 1. After surgery, the animals were placed on a heating pad in a humidity- and temperature-controlled chamber. Bladders were manually evacuated twice daily until autonomous urination was established. The rats were euthanized by transcardiac perfusion at 7- and 56-days post-SCI.

\section{US stimulation system}

The stereotactic frame-based US stimulation system (Figure 1a) was composed of 3D-printed rat holders, a coupling water tank, a flat piezoelectric single-element transducer (A303S, $\mathrm{f}=1 \mathrm{MHz}, 12.7-\mathrm{mm}$ diameter, Olympus America Inc., Waltham, MA, USA), a position XYZ controller (51900; Stoelting Inc., Wood Dale, IL, USA), a function/arbitrary waveform generator (33220A; Agilent Technologies, Santa Clara, CA, USA), and an RF power amplifier (A-150; Electronics \& Innovation, Rochester, NY, USA). The center frequency of the transducer was $1 \mathrm{MHz}$. The US beam profile on the free field was measured using an acoustic intensity measurement system (AIMS III; ONDA, Sunnyvale, CA, USA) with a hydrophone (HGL-400; ONDA; Figure $\mathrm{S} 1)$. The transducer adaptor apparatus was designed considering the beam profile, with a height of $30 \mathrm{~mm}$, the focus point of ultrasonic energy.

\section{Experimental setup and US stimulation}

Following anesthetization, SCI rats were placed on a 3D-printed rat holder (Figure 1a). The US transducer was moved to the target position using an XYZ controller. The Z-axis, a constant distance between the transducer 
and the stimulation point, was adjusted using an inverted triangular 3D-printed transducer adaptor that clipped onto the transducer and allowed targeting. After obtaining the coordinates, the acquired values were set to zero. The water tank was placed at the target point of the dorsal laminectomy site after application of the ultrasonic gel. The transducer was positioned with its active element immersed in water, approximately $30 \mathrm{~mm}$ from the target region. All animals were subjected to two different stimulation conditions. The following pulse US parameters were used: (1) SCIU5, acoustic frequency of $1 \mathrm{MHz}$, pulse repetition frequency (PRF) of $1 \mathrm{kHz}, 5 \%$ duty cycle, acoustic power of $0.8 \mathrm{~W} / \mathrm{cm}^{2}$ and (2) SCIU40, acoustic frequency of $1 \mathrm{MHz}$, PRF of $1 \mathrm{kHz}, 40 \%$ duty cycle, and acoustic power of $0.8 \mathrm{~W} / \mathrm{cm}^{2}$. The total sonication time was $5 \mathrm{~min}$ (Table 2). These US parameters were based on the neuromodulation parameter ranges for excitatory or suppressive responses ${ }^{49}$.

\section{Experimental design}

US stimulation was applied for 5 min daily from day 2 to day 4 ( 3 times total). The experimental protocol was divided into short-term ( 7 total) or long-term ( 8 weeks; Figure 1b). The effects of US on locomotion and inflammation were assessed after SCI in three randomly assigned treatment groups: 1) sham (SCI only), 2) SCIU5 $(\mathrm{SCI}+\mathrm{US}$ sonication, duty cycle $5 \%$ ), and 3$)$ SCIU40 (SCI + US sonication, duty cycle $40 \%)$. The detailed experimental groups and US parameters are described in Table 2.

MR imaging

Images of rats were obtained 14 days post-injury. All the T-spine MRI scans were conducted on a $3 \mathrm{~T}$ clinical dedicated head MR scanner (Siemens Magnetom Skyra; Siemens Medical Solutions, Erlangen, Germany). A 40 mm loop-type RF coil was used, and rats were placed in the prone position under the coil housing. Sagittal images of the spine were acquired using 2D turbo spin-echo T2-weighted images. The acquisition parameters were as follows: field of view $=30 \times 30 \mathrm{~mm}$; matrix size $=128 \times 128$; number of slices $=10$; slice thickness $=0.7 \mathrm{~mm}$; no gap; repetition time $=2500 \mathrm{~ms}$; echo time $=101 \mathrm{~ms}$; number of averages $=20$; echo train length $=10$. MR images were assessed using Image J 1.52 (National Institutes of Health, Bethesda, MD, USA).

\section{Evaluation of locomotor function}

Locomotor recovery after SCI was evaluated using the open-field BBB scale and ladder rung test. Two observers, blinded to the US treatment groups, evaluated animals based on the BBB open-field locomotion rating scale. The 21-point open-field locomotion score is based on the hindlimb locomotor ability of the SCI model and 
reflects the early (BBB score $0-7)$, intermediate (8-13), and late phases (14-21) of recovery ${ }^{50}$. After SCI rats briefly adapted to the conditions, they were observed in an open field for $5 \mathrm{~min}$. consisted of sidewalls (length: $1 \mathrm{~m}$, height: $50 \mathrm{~cm}$ ) and metal rungs (diameter: $3 \mathrm{~mm}$, length: $150 \mathrm{~mm}$ ). Mistakes during walking on irregular runways were evaluated. During pretraining, the animals passed across the horizontal ladder twice. After familiarization, the SCI rats were recorded using a video camera. The hindlimb foot error was defined as a complete miss or slight/deep slip, and errors were counted. If an SCI rat could not take a step, it was assigned one error per bar, resulting in a total of 35 errors. All animals showed almost complete paraplegia $1 \mathrm{~d}$ after SCI. After the surgery, motor function was assessed on days 3, 5, and 7, then weekly for 8 weeks.

\section{Tissue preparation and histology}

The rats were transcardially perfused with $100 \mathrm{~mL}$ of fresh $4 \%$ paraformaldehyde (PFA) in $1 \%$ phosphatebuffered saline (PBS; pH 7.4), followed by $100 \mathrm{~mL}$ of $1 \%$ PBS 7 days or 8 weeks after SCI. The injured spinal cords (approximately $6 \mathrm{~mm}$ ) were dissected and post-fixed in PFA for $24 \mathrm{~h}$ at $4{ }^{\circ} \mathrm{C}$. The tissues were embedded in paraffin blocks, and coronal sections were obtained. For H\&E staining, the spinal cords were embedded in paraffin and serially sectioned at a 5 - $\mu \mathrm{m}$-thick coronal plane. The tissue sections were deparaffinized with xylene, rehydrated in a series of ethanol $(100 \%, 95 \%, 70 \%$, and $50 \%)$, washed with distilled water (DW), and stained with H\&E every $60^{\text {th }}$ section ( $250 \mu \mathrm{m}$ apart).

\section{Fluoro-Jade C (FJC) staining}

FJC staining was performed by optimizing the Ready-to-Dilute FJC Staining Kit (Biosensis, Tebarton, South Australia), according to the manufacturer's protocol ${ }^{52}$. Following deparaffination, the sections were incubated in a potassium permanganate solution (1:15) in DW for 15 min and subsequently rinsed in DW. Samples were then incubated in FJC solution (1:50) with DW for 30 min. Coverslips were then added with a fluorescence mounting medium (Dako, Glostrup, Denmark). The tissues were counterstained with DAPI (Dako).

\section{Immunofluorescence analysis}

Next, $5-\mu$ m-thick transverse sections were blocked with PBS containing $1 \%$ BSA, $3 \%$ normal goat serum, and $0.4 \%$ Triton $\mathrm{X}-100$ for $1 \mathrm{~h}$ at room temperature following the antigen retrieval process. Tissues were incubated 
Kenilworth, NJ, USA), iNOS (1:200; \#ab15323, Abcam, Cambridge, UK), and TNF- $\alpha 1$ (200; \#ab6671; Abcam). Alexa Fluor 488-labeled goat anti-mouse-IgG (1:1000; \#A32723, Invitrogen, Carlsbad, CA, USA), and Alexa Fluor 633-labeled goat anti-rabbit-IgG (1:1000; \#A32731, Invitrogen) were used as secondary antibodies. After immunolabeling, the tissue slides were mounted in DAPI-containing fluorescence mounting medium (Dako).

\section{Image analysis}

All tissue slides were captured using a Zeiss Axio Scan.Z1 Digital Slide Scanner (Carl Zeiss, Oberkochen, Germany) using a $20 \times$ objective lens. Five slices stained with H\&E from each animal were used to assess the lesion cavity volume. To evaluate tissue density, 4-6 regions of interest (ROIs) per slide were selected and analyzed at high magnification $(\times 200)$. When selecting ROIs, areas except for the necrotic zone were obtained in the H\&E-stained images to determine the tissue density resulting from hemorrhage and edema due to extensive spinal cord damage. To quantify immunofluorescence intensity, five serial sections in the transverse plane from each animal were analyzed using ImageJ (version 1.40; National Institutes of Health, Bethesda, MD, USA). Fluorescence intensity was defined as the relative change in fluorescence compared to that in the sham control and is presented as a fold change.

\section{Western blotting}

The SCI site was extracted and homogenized in RIPA lysis buffer containing protease inhibitor (150 mM sodium chloride, $0.1 \%$ SDS, $1 \%$ Triton $\mathrm{X}-100$, $1 \%$ sodium deoxycholate, and $50 \mathrm{mM}$ Tris-HCl). After centrifugation, the supernatant was transferred to a new tube. Protein concentration was determined using the Pierce BCA Protein Assay Kit (Thermo Fisher Scientific, Waltham, MA, USA). The proteins (40 $\mu \mathrm{g} / \mathrm{lane})$ were separated by $8-20 \%$ SDS-PAGE and transferred electrophoretically to a polyvinylidene difluoride membrane (Millipore, Billerica, MA, USA). After blocking with 5\% skim milk, the membranes were incubated with an antiiNOS mouse monoclonal antibody (1:200; \#MAB9502, R\&D Systems, Minneapolis, MN, USA), anti-TNF- $\alpha$ rat polyclonal antibody (1:500; \#ARC3012, Invitrogen), anti-ED-1 mouse monoclonal antibody (1:200; \#MAB1435, Merck), and anti-GAPDH antibody (1:10,000; \#88845, Cell Signaling, Danvers, MA, USA), and subsequently incubated with the HRP-conjugated anti-rabbit-IgG secondary antibody (1:3,000; \#7074, Cell Signaling), and anti-rat-IgG secondary antibody (1:3,000; \#7077, Cell Signaling) for $2 \mathrm{~h}$ at room temperature. Signaling was detected using a chemiluminescence kit (Amersham Pharmacia Biotech Inc., Piscataway, NJ, USA). GAPDH was used as the loading control. 
All data are presented as means \pm SEM. Statistical analyses and data visualization were performed using

330 Prism version 6 (GraphPad Software, Inc., La Jolla, CA, USA). One- or two-way ANOVA and post-hoc Tukey's

331 multiple comparison tests were used for statistical analyses. Statistical significance was set at $P<0.05$. 


\section{References}

3331 Dumont, R. J. et al. Acute spinal cord injury, part I: pathophysiologic mechanisms. Clinical 334 neuropharmacology 24, 254-264 (2001).

3352 Tator, C. H. \& Fehlings, M. G. Review of the secondary injury theory of acute spinal cord trauma with 336 emphasis on vascular mechanisms. Journal of neurosurgery 75, 15-26 (1991).

3373 Tator, C. H. Update on the pathophysiology and pathology of acute spinal cord injury. Brain pathology $338 \quad 5,407-413(1995)$.

3394 Ahuja, C. S. et al. Traumatic spinal cord injury-repair and regeneration. Neurosurgery 80, S9-S22 $340 \quad$ (2017).

3415 Kim, Y.-H., Ha, K.-Y. \& Kim, S.-I. Spinal cord injury and related clinical trials. Clinics in orthopedic $342 \quad$ surgery 9, 1-9 (2017).

3436 Ahuja, C. S. et al. Traumatic Spinal Cord Injury-Repair and Regeneration. Neurosurgery 80, S9-s22, 344 doi:10.1093/neuros/nyw080 (2017).

3457 Casha, S. et al. Results of a phase II placebo-controlled randomized trial of minocycline in acute spinal $346 \quad$ cord injury. Brain 135, 1224-1236 (2012).

3478 Nagoshi, N., Nakashima, H. \& Fehlings, M. G. Riluzole as a neuroprotective drug for spinal cord injury: 348 from bench to bedside. Molecules 20, 7775-7789 (2015).

3499 Vink, R. Magnesium in the CNS: recent advances and developments. Magnesium Research 29, 95-101 $350 \quad$ (2016).

Anna, Z. et al. Therapeutic potential of olfactory ensheathing cells and mesenchymal stem cells in spinal cord injuries. Stem cells international 2017 (2017). transplanted adult neural stem/progenitor cells, chondroitinase, and growth factors promote functional repair and plasticity of the chronically injured spinal cord. Journal of Neuroscience 30, 1657-1676 (2010). Theodore, N. et al. First human implantation of a bioresorbable polymer scaffold for acute traumatic spinal cord injury: a clinical pilot study for safety and feasibility. Neurosurgery 79, E305-E312 (2016). Biomedical Materials Research Part A 106, 698-705 (2018). 

implants after spinal cord injury. Bioelectronic Medicine 5, 10 (2019). Taccola, G., Sayenko, D., Gad, P., Gerasimenko, Y. \& Edgerton, V. And yet it moves: recovery of volitional control after spinal cord injury. Progress in neurobiology 160, 64-81 (2018). 28, 49-62 (2017). Anderson, K. D. et al. Safety of autologous human schwann cell transplantation in subacute thoracic spinal cord injury. Journal of neurotrauma 34, 2950-2963 (2017).

Corotchi, M. C., Popa, M. A. \& Simionescu, M. Testosterone stimulates proliferation and preserves stemness of human adult mesenchymal stem cells and endothelial progenitor cells. Rom J Morphol Embryol 57, 75-80 (2016).

Speed, C. Therapeutic ultrasound in soft tissue lesions. Rheumatology 40, 1331-1336 (2001).

Wood, R. W. \& Loomis, A. L. XXXVIII. The physical and biological effects of high-frequency soundwaves of great intensity. The London, Edinburgh, and Dublin philosophical magazine and journal of science 4, 417-436 (1927).

Harvey, E. N. Biological aspects of ultrasonic waves, a general survey. The Biological Bulletin 59, 306325 (1930).

Kulke, M. H. et al. Evolving diagnostic and treatment strategies for pancreatic neuroendocrine tumors. Journal of hematology \& oncology 4, 29 (2011). 450 (Springer, 2016).

Wood, A. K. \& Sehgal, C. M. A review of low-intensity ultrasound for cancer therapy. Ultrasound in medicine \& biology 41, 905-928 (2015).

Chowdhury, S. M., Lee, T. \& Willmann, J. K. Ultrasound-guided drug delivery in cancer. Ultrasonography 36, 171 (2017).

Miller, D. L. et al. Overview of therapeutic ultrasound applications and safety considerations. Journal of ultrasound in medicine 31, 623-634 (2012). 398 (Thieme Medical Publishers). 

sialadenitis. Arthritis research \& therapy 17, 1-12 (2015). Nagao, M. et al. LIPUS suppressed LPS-induced IL-1 $\alpha$ through the inhibition of NF-kB nuclear translocation via AT1-PLC $\beta$ pathway in MC3T3-E1 cells. Journal of cellular physiology 232, 3337-3346 (2017).

Yang, Q. et al. Low-intensity ultrasound-induced anti-inflammatory effects are mediated by several new mechanisms including gene induction, immunosuppressor cell promotion, and enhancement of exosome biogenesis and docking. Frontiers in Physiology 8, 818 (2017). Chung, J.-I. et al. Anti-inflammatory effect of low intensity ultrasound (LIUS) on complete Freund's adjuvant-induced arthritis synovium. Osteoarthritis and cartilage 20, 314-322 (2012). permeability induced by carrageenan injection in rats. Journal of Inflammation 17, 1-8 (2020). Ito, A. et al. Ultrasound therapy with optimal intensity facilitates peripheral nerve regeneration in rats through suppression of pro-inflammatory and nerve growth inhibitor gene expression. PloS one 15, e0234691 (2020). communications 10, 1-10 (2019). on memory impairment and brain damage in a rat model of vascular dementia. Radiology 282, 113-122 (2017). and histological outcomes after experimental traumatic brain injury. Scientific reports 7, 1-10 (2017). Takeoka, A., Vollenweider, I., Courtine, G. \& Arber, S. Muscle spindle feedback directs locomotor recovery and circuit reorganization after spinal cord injury. Cell 159, 1626-1639, doi:10.1016/j.cell.2014.11.019 (2014).

41438 de Oliveira Perrucini, P. D. et al. Anti-Inflammatory and Healing Effects of Pulsed Ultrasound Therapy on Fibroblasts. American journal of physical medicine \& rehabilitation 99, 19-25, doi:10.1097/phm.0000000000001265 (2020).

41739 Zhou, S. et al. Low intensity pulsed ultrasound accelerates macrophage phagocytosis by a pathway that 
requires actin polymerization, Rho, and Src/MAPKs activity. Cellular signalling 20, 695-704, doi:10.1016/j.cellsig.2007.12.005 (2008).

$42040 \quad$ Su, W. S., Wu, C. H., Chen, S. F. \& Yang, F. Y. Transcranial ultrasound stimulation promotes brainderived neurotrophic factor and reduces apoptosis in a mouse model of traumatic brain injury. Brain stimulation 10, 1032-1041, doi:10.1016/j.brs.2017.09.003 (2017).

Kim, K. H. et al. Low-intensity ultrasound attenuates paw edema formation and decreases vascular permeability induced by carrageenan injection in rats. Journal of inflammation (London, England) 17, 7, doi:10.1186/s12950-020-0235-x (2020).

Chung, J. I. et al. Anti-inflammatory effect of low intensity ultrasound (LIUS) on complete Freund's

20, 314-322, doi:10.1016/j.joca.2012.01.005 (2012).

Galtrey, C. M. \& Fawcett, J. W. The role of chondroitin sulfate proteoglycans in regeneration and plasticity in the central nervous system. Brain research reviews

54, 1-18, doi:10.1016/j.brainresrev.2006.09.006 (2007).

Kong, X. \& Gao, J. Macrophage polarization: a key event in the secondary phase of acute spinal cord injury. Journal of cellular and molecular medicine 21, 941-954, doi:10.1111/jcmm.13034 (2017).

Kim, H. et al. Suppression of EEG visual-evoked potentials in rats through neuromodulatory focused ultrasound. Neuroreport 26, 211-215, doi:10.1097/wnr.0000000000000330 (2015). $15.2016(2016)$.

Dumont, R. J. et al. Acute spinal cord injury, part I: pathophysiologic mechanisms. Clinical neuropharmacology 24, 254-264, doi:10.1097/00002826-200109000-00002 (2001). spinal cord injury: characterization of a force-defined injury device. Journal of neurotrauma 20, 179193 (2003).

Michael, P., Eitan, K. \& Shy, S. Cell-type-selective effects of intramembrane cavitation as a unifying theoretical framework for ultrasonic neuromodulation 123. Eneuro. 3, 1-16 (2016).

Basso, D. M., Beattie, M. S. \& Bresnahan, J. C. A sensitive and reliable locomotor rating scale for open 
$44851 \quad$ Metz, G. A. \& Whishaw, I. Q. Cortical and subcortical lesions impair skilled walking in the ladder rung walking test: a new task to evaluate fore-and hindlimb stepping, placing, and co-ordination. Journal of neuroscience methods 115, 169-179 (2002).

52 Yang, L.-Y. et al. Post-trauma administration of the pifithrin- $\alpha$ oxygen analog improves histological and functional outcomes after experimental traumatic brain injury. Experimental neurology 269, 56-66 (2015).

Acknowledgments: This research was supported by the Brain Research Program through the National Research Foundation of Korea (NRF), funded by the Ministry of Science, ICT \& Future Planning [grant numbers NRF2016M3C7A1913933, NRF-2019M3E5D1A02069399, and NRF-2019R1C1C1011615]. The authors thank Changzhu Jin, Mi-Jeong Kim, Ye Jin Seo, Byeongjin Jung, and Hyo Jin Choi for developing the methodology and technical support.

460 Author contributions: K. Kim and J. Park contributed to the study conception and design. Y. Hong, E. Lee, K. 461 Park, Mun Han developed the methodology. Data acquisition was performed by Y. Hong, and M. Han. The data 462 were analyzed and interpreted by Y. Hong, E. Lee, M. Han, K. Kim, and J. Park. Writing, review, and/or revision 463 of the manuscript were performed by Y. Hong, E. Lee, M. Han, K. Kim, and J. Park. Administrative, technical, 464 and material support was provided by Y. Hong, E. Lee, M. Han, K. Kim, and J. Park. K. Kim and J. Park supervised 465 the study.

466 Competing interests: The authors declared no competing interests.

467 Data availability: Data are available from the corresponding author upon reasonable request 
Figure 1. Experimental setup of the customized US stimulation system. (A) US stimulation with two different parameters, SCIU5 and SCIU40, was applied to spinal cord injury model rats. Schematic illustration of the customized US stimulation systems. (B) Experimental design for the evaluation of the effect of US stimulation on a rat model of SCI. Models were established on day 0 and subjected to US stimulation three times $(2,3$, and 4 days following traumatic cord injury. Protocol 1; acute SCI phase study (days 1-7). Protocol 2; chronic SCI phase study (8 weeks).

476

Figure 2. Improved function and reduced tissue damage in the acute spinal cord injury (SCI) phase. (a) The Basso, Beattie, and Bresnahan (BBB) score for hindlimb locomotor function was evaluated at 3, 5, and 7 days after US treatment post-SCI (black line indicates the sham group, $\mathrm{n}=21$; red line indicates the SCIU5 group, $\mathrm{n}=$ 21; blue line indicates the SCIU40 group, $\mathrm{n}=18$ ). (b) Fine motor coordination was evaluated using the ladder rung test by counting error steps. An error was defined as a slip, miss, or drag. (c, d) Representative images of spinal cord transverse sections for H\&E staining from each group and enlarged images. (e) Quantification of the cavity area following contusion injury ( 5 slices per rat, $n=3$ ). (f) The tissue density was assessed in H\&E-stained slices selected from four regions of interest per spinal cord tissue in five slices from each of three rats, excluding the necrotic zone. (g) FJC staining and enlarged images at high magnification $(\times 200$, red square box $)$. (h, i) Quantitative analysis of FJC-positive cells and intensity based on mean cell counts from six regions of interest per spinal cord tissue in five slices from each of three rats. Data are expressed as means $\pm \mathrm{SEM}$. Two-way ANOVA with Turkey's tests for the multiple comparisons was used. ${ }^{*} P<0.05$, ${ }^{* *} P<0.01,{ }^{* * *} P<0.001$, and ${ }^{* * * *} P<$ 0.0001 compared with the sham control, ${ }^{\# \#} P<0.01,{ }^{\# \#} P<0.001,{ }^{\# \# \# P} P<0.0001$ compared with the SCIU5-treated group.

Figure 3. Ultrasound (US) stimulation reduces ED-1, iNOS, and TNF- $\alpha$ expression in the acute spinal cord injury (SCI) phase. (a-c) ED-1 (macrophage/microglia, green) and iNOS (inducible nitric oxide synthase, red) were evaluated in the sham (a), SCIU40 (b), and SCIU5 (c) experimental groups at 7 days post-SCI (five sections per rat, $\mathrm{n}=3$ ). $(\mathbf{d}-\mathbf{f})$ Cross-sections were counterstained with anti-TNF- $\alpha$ (red) and ED-1 (green) antibodies (five sections per rat, $\mathrm{n}=3$ ). (g-i) Quantification of fluorescence intensity of ED-1, iNOS, and TNF- $\alpha$. Two-way 
with the sham control, ${ }^{\#} P<0.05,{ }^{\# \#} P<0.001,{ }^{\# \# \#} P<0.0001$ compared with the SCIU5-treated group. (j) Representative western blotting results for ED-1, iNOS, TNF- $\alpha$, and GAPDH at 7 days post-SCI (Lane 1, sham; lane 2, SCIU40; lane 3, SCIU5;). Relative expression levels of ED-1, iNOS, and TNF- $\alpha$ were calculated after normalization to the level of GAPDH. Data are presented as means \pm SEM. Two-tailed Student's $t$-tests were used for comparison. ${ }^{*} P<0.05$ compared with the sham control.

\section{Figure 4. Ultrasound (US) stimulation significantly improves motor function and suppresses tissue damage} in the chronic spinal cord injury (SCI) phase. (a) Basso, Beattie, and Bresnahan (BBB) scores for hindlimb locomotor function evaluated 7 days before (-7) and 1-8 weeks after US stimulation post-SCI (black line indicates the sham group, $\mathrm{n}=7$; red line indicates the SCIU5 group, $\mathrm{n}=8$; blue line indicates the SCIU40 group, $\mathrm{n}=7$ ). (b) Fine motor coordination was evaluated using the ladder rung test based on the percentage of errors, defined as a slip, miss, or drag. (c, d) Transverse spinal cord sections obtained 8 weeks after SCI were stained with H\&E to evaluate the area of tissue injury and cavity. The injured cavity area is marked with a dashed red line, and the red box in C is magnified $20 \times$ in D. (e) A total of five slices from each of three rats were selected, and the cavity area following contusion injury was quantified. (f) Tissue density was assessed using H\&E-stained slices based on four regions of interest per spinal cord tissue in five slices from each of three rats, excluding the necrotic zone. (g) FJC staining of lesion areas in transverse sections of the spinal cord and the enlarged images at high magnification $(\times 200$, red square box $)$. (h, i) FJC staining was evaluated quantitatively based on the number of FJC/DAPI co-labeled cells and fluorescence intensity in six regions of interest per spinal cord tissue in five slices from each of three rats. Data are expressed as means \pm SEM. Two-way ANOVA and Turkey's tests for the multiple comparisons were used. ${ }^{*} P<0.05,{ }^{* * *} P<0.001,{ }^{* * * *} P<0.0001$ compared with the sham control, ${ }^{\#} P<0.05$,

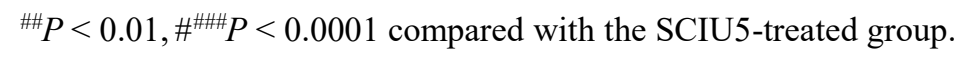


Tables:

524

Table 1. Infinite Horizon impact parameters

\begin{tabular}{lllll}
\hline & Force (Kdyn) & Velocity $(\mathrm{mm} / \mathrm{s})$ & Time $(\mathrm{ms})$ & Animal number \\
\hline 140 Kdyn & $140 \pm 7$ & $122 \pm 5$ & 0 & 7 \\
150 Kdyn & $152 \pm 2$ & $126 \pm 2$ & 0 & 8 \\
200 Kdyn & $214 \pm 20$ & $119 \pm 5$ & 0 & 8 \\
\hline
\end{tabular}

525

526 Table 2. Summary of the experimental ultrasound (US) parameters

\begin{tabular}{|c|c|c|c|c|c|c|c|}
\hline Protocol & Group & $\begin{array}{l}\text { US } \\
\text { treatment } \\
\text { condition }\end{array}$ & $\begin{array}{l}\text { Center } \\
\text { frequency } \\
(\mathrm{MHz})\end{array}$ & $\begin{array}{l}\text { Pulse } \\
\text { repetition } \\
\text { frequency } \\
(\mathrm{kHz})\end{array}$ & $\begin{array}{l}\text { Acoustic } \\
\text { Intensity } \\
\left(\mathrm{W} / \mathrm{cm}^{2}\right)\end{array}$ & $\begin{array}{l}\text { Duty } \\
\text { Cycle } \\
(\%)\end{array}$ & $\begin{array}{l}\text { Animal } \\
\text { number }\end{array}$ \\
\hline \multirow{3}{*}{1} & 1 & Sham & - & - & - & - & 7 \\
\hline & 2 & SCIU5 & 1 & 1 & 0.8 & 5 & 8 \\
\hline & 3 & SCIU40 & 1 & 1 & 0.8 & 40 & 7 \\
\hline \multirow{3}{*}{2} & 1 & Sham & - & - & & - & 21 \\
\hline & 2 & SCIU5 & 1 & 1 & 0.8 & 5 & 21 \\
\hline & 3 & SCIU40 & 1 & 1 & 0.8 & 40 & 18 \\
\hline
\end{tabular}

527

528 


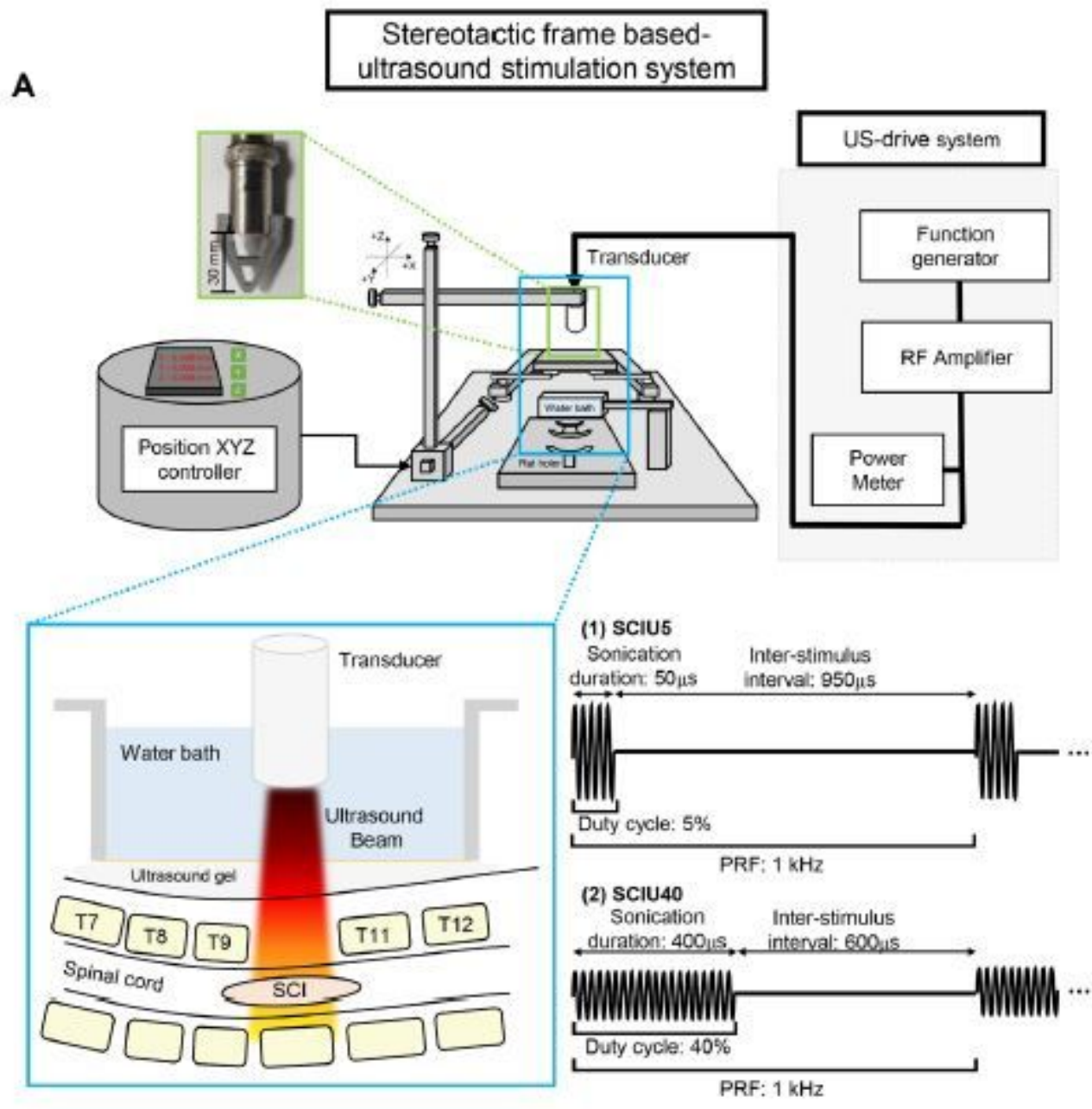

B

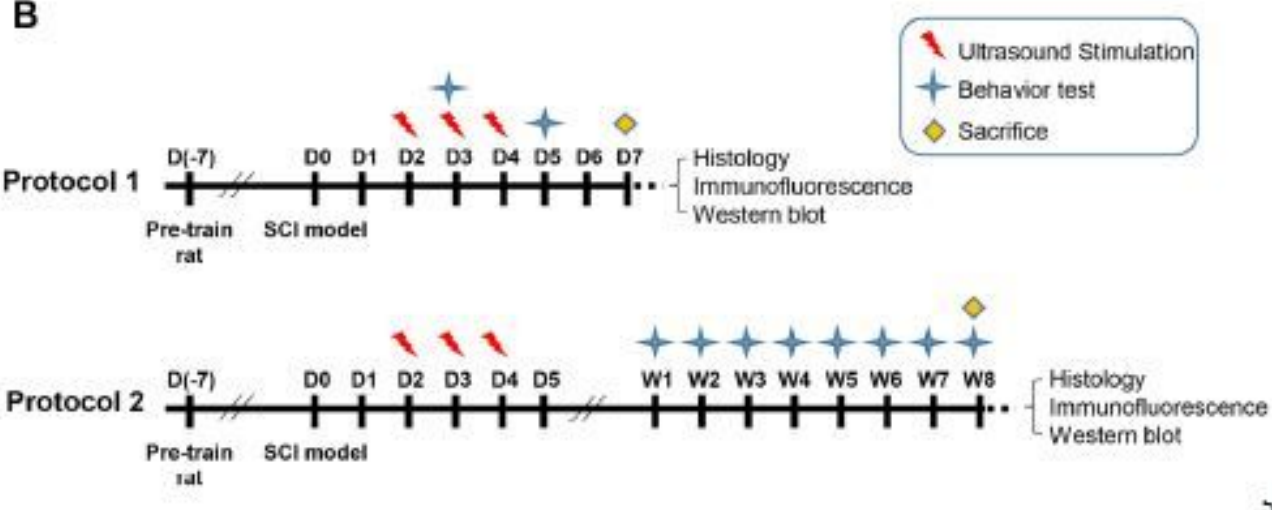

Figure 1

Experimental setup of the customized US stimulation system. (A) US stimulation with two different parameters, SCIU5 and SCIU40, was applied to spinal cord injury model rats. Schematic illustration of the customized US stimulation systems. (B) Experimental design for the evaluation of the effect of US 
stimulation on a rat model of $\mathrm{SCl}$. Models were established on day 0 and subjected to US stimulation three times $(2,3$, and 4 days following traumatic cord injury. Protocol 1; acute SCl phase study (days 17). Protocol 2; chronic SCI phase study (8 weeks).

A

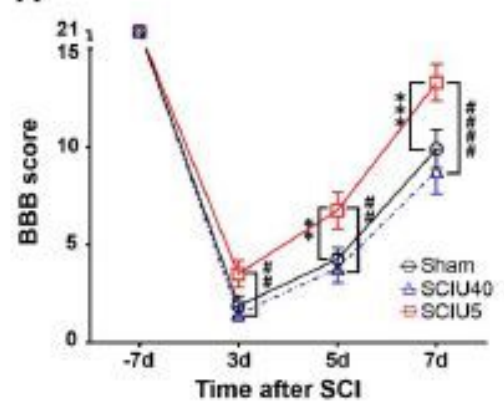

C

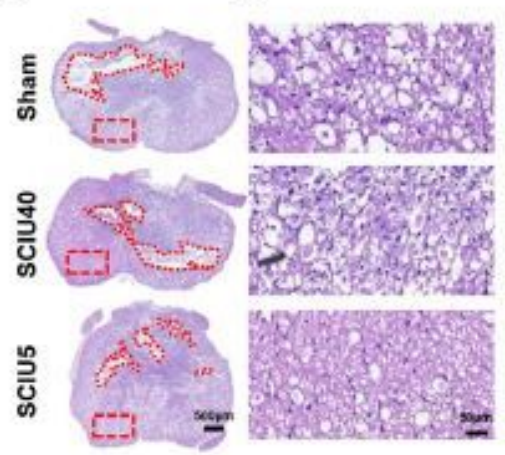

$\mathbf{E}$

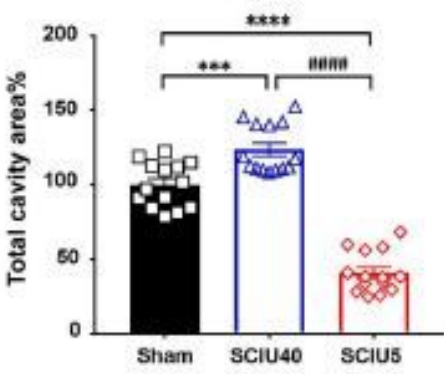

$\mathbf{F}$

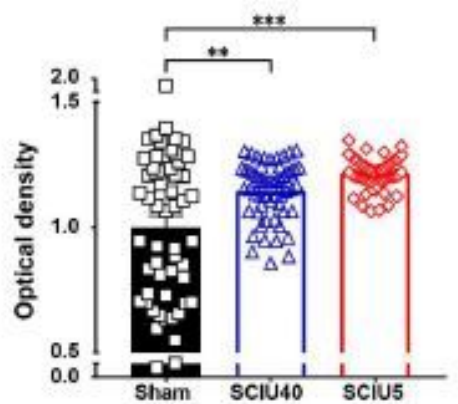

B

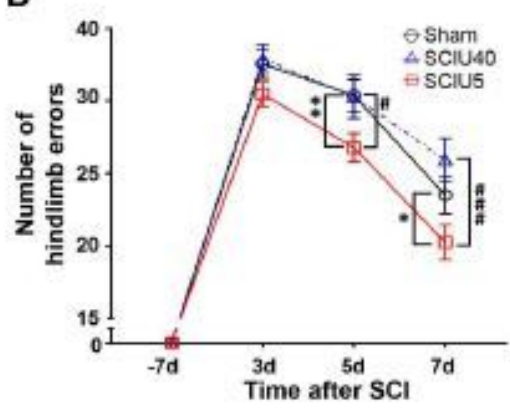

G
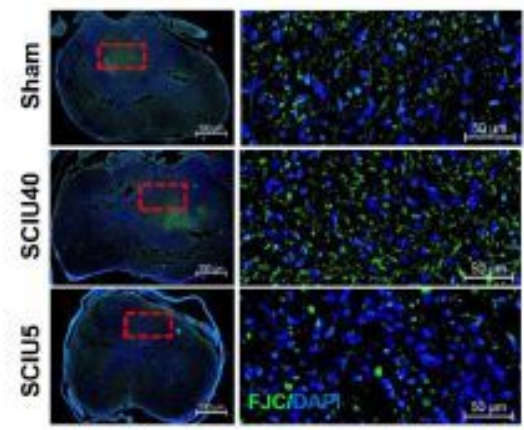

H

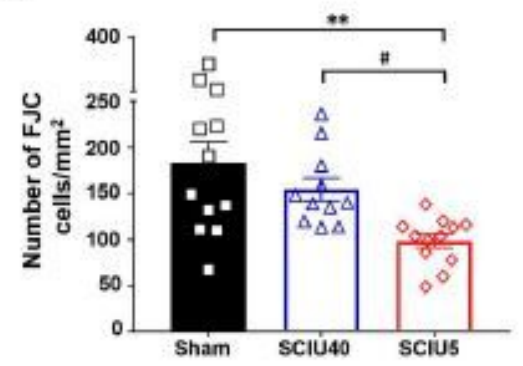

I

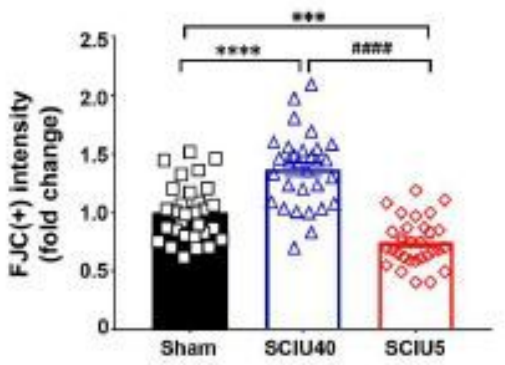

Figure 2

Improved function and reduced tissue damage in the acute spinal cord injury (SCl) phase. (a) The Basso, Beattie, and Bresnahan (BBB) score for hindlimb locomotor function was evaluated at 3,5 , and 7 days 
after US treatment post-SCI (black line indicates the sham group, $n=21$; red line indicates the SCIU5 group, $n=21$; blue line indicates the SCIU40 group, $n=18$ ). (b) Fine motor coordination was evaluated using the ladder rung test by counting error steps. An error was defined as a slip, miss, or drag. (c, d) Representative images of spinal cord transverse sections for H\&E staining from each group and enlarged images. (e) Quantification of the cavity area following contusion injury ( 5 slices per rat, $n=3$ ). (f) The tissue density was assessed in H\&E-stained slices selected from four regions of interest per spinal cord tissue in five slices from each of three rats, excluding the necrotic zone. (g) FJC staining and enlarged images at high magnification ( $\times 200$, red square box). ( $h, i)$ Quantitative analysis of FJC-positive cells and intensity based on mean cell counts from six regions of interest per spinal cord tissue in five slices from each of three rats. Data are expressed as means \pm SEM. Two-way ANOVA with Turkey's tests for the multiple comparisons was used. ${ }^{*} P<0.05, * \star P<0.01$, ${ }^{\star \star \star} P<0.001$, and ${ }^{*} \star \star * P<0.0001$ compared with the sham control, \#\#P $<0.01$, \#\#\#P $<0.001$, \#\#\#\#P $<0.0001$ compared with the SCIU5-treated group. 

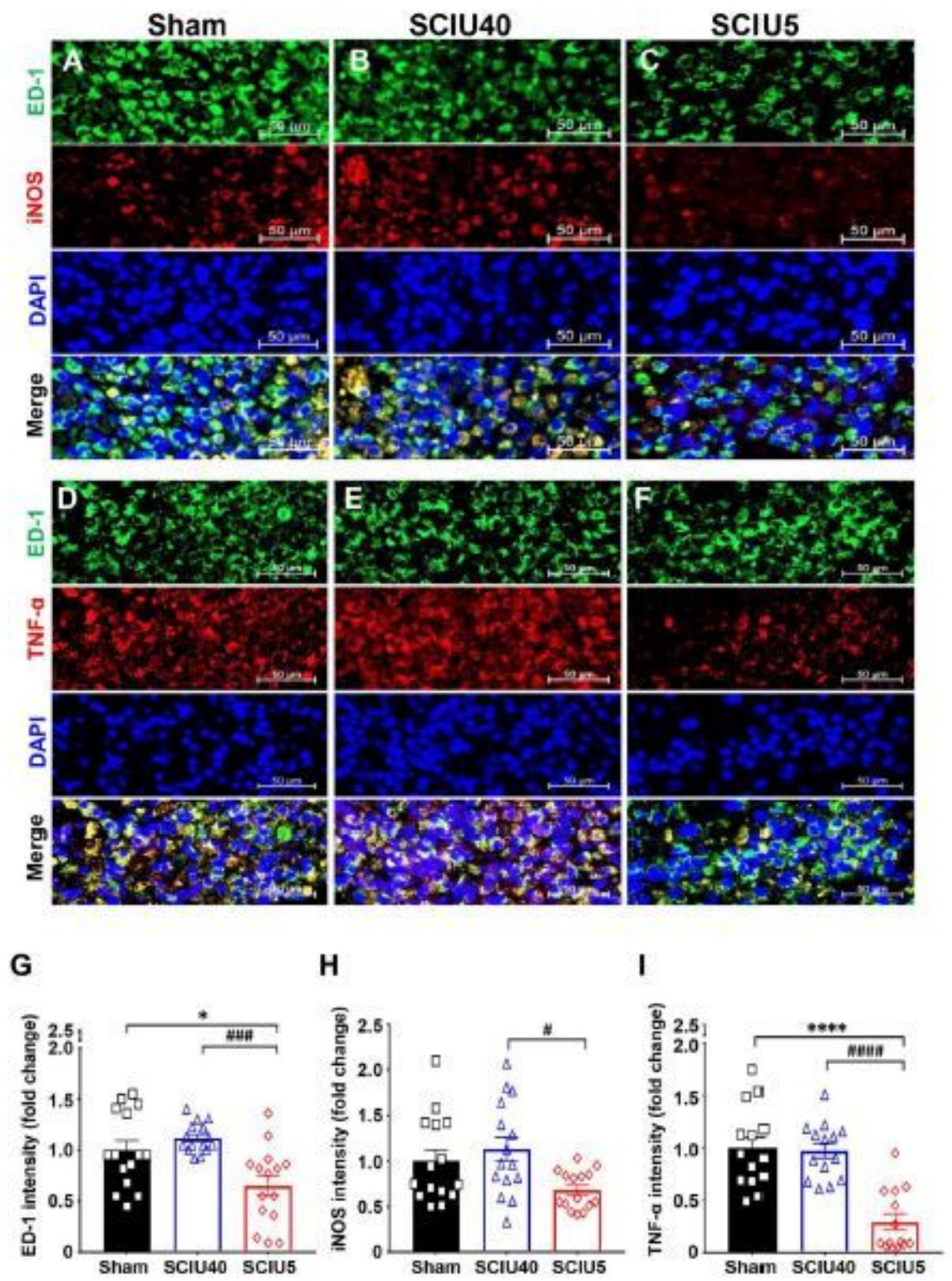

$\mathbf{J}$

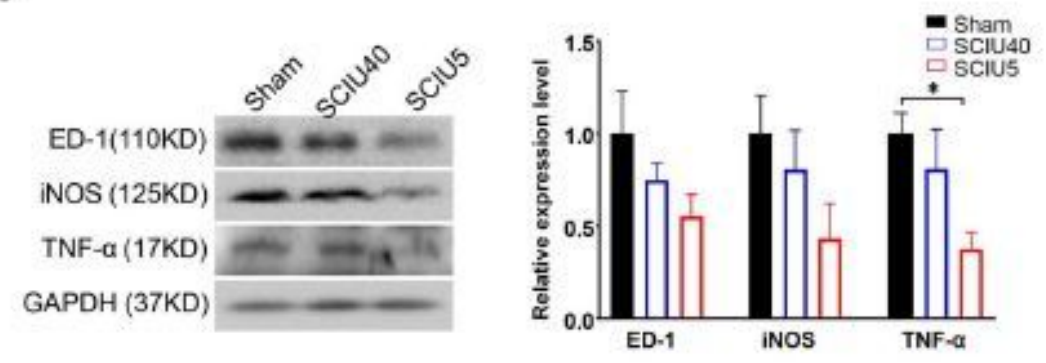

\section{Figure 3}

Ultrasound (US) stimulation reduces ED-1, iNOS, and TNF-a expression in the acute spinal cord 491 injury (SCl) phase. (a-c) ED-1 (macrophage/microglia, green) and iNOS (inducible nitric oxide synthase, red) were evaluated in the sham (a), SCIU40 (b), and SCIU5 (c) experimental groups at 7 days post-SCI (five sections 493 per rat, $n=3)$. ( $(d-f)$ Cross-sections were counterstained with anti-TNF-a (red) and ED-1 (green) antibodies (five 494 sections per rat, $n=3$ ). ( $g-i)$ Quantification of fluorescence intensity of ED-1, 
iNOS, and TNF-a. Two-way ANOVA with Turkey's test for multiple comparisons was used for analyses. *P

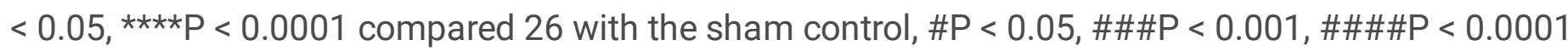
compared with the SCIU5-treated group. (j) Representative western blotting results for ED-1, iNOS, TNF-a, and GAPDH at 7 days post-SCI (Lane 1, sham; lane 2, SCIU40; lane 3, SCIU5;). Relative expression levels of ED-1, iNOS, and TNF-a were calculated after normalization to the level of GAPDH. Data are presented as means \pm SEM. Two-tailed Student's t-tests were used for comparison. ${ }^{*} \mathrm{P}<0.05$ compared with the sham control.

A

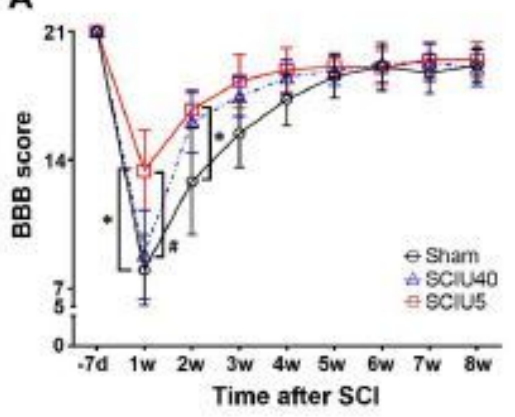

C D

黄

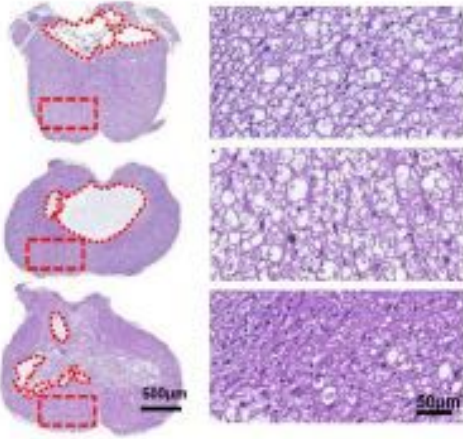

E

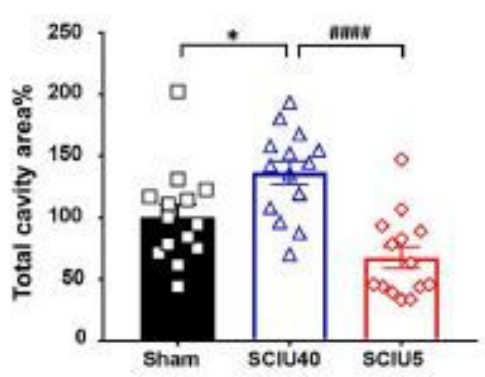

F

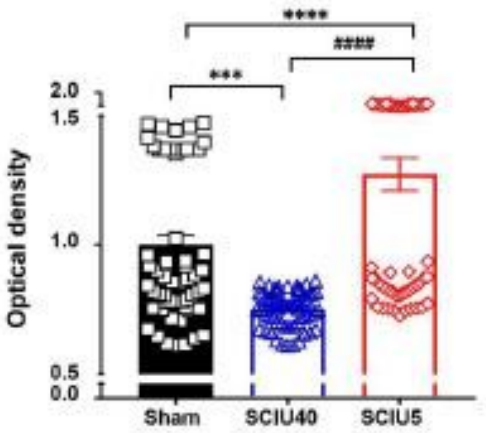

B

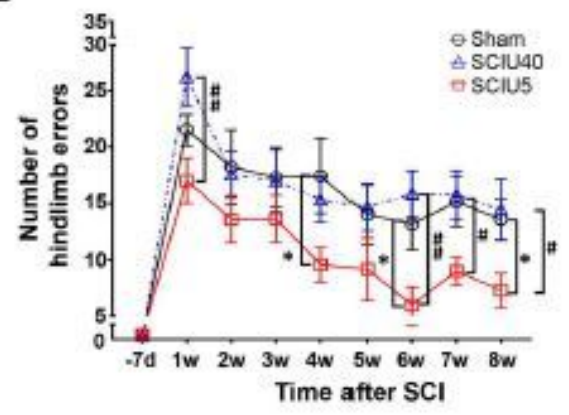

G
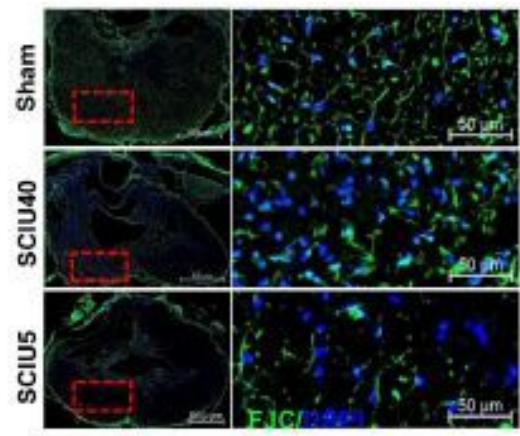

H

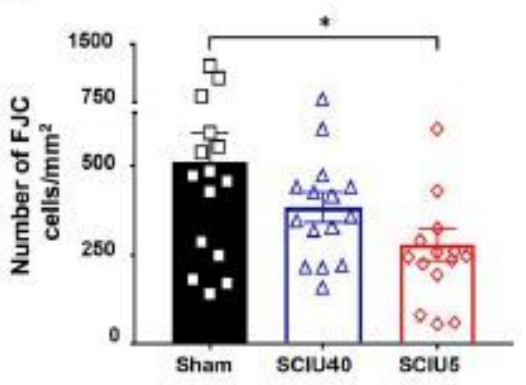

I

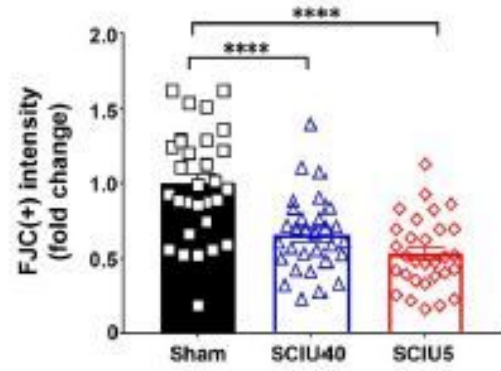




\section{Figure 4}

Ultrasound (US) stimulation significantly improves motor function and suppresses tissue damage in the chronic spinal cord injury (SCl) phase. (a) Basso, Beattie, and Bresnahan (BBB) scores for hindlimb 503 locomotor function evaluated 7 days before $(-7)$ and $1-8$ weeks after US stimulation post-SCl (black line indicates the sham group, $n=7$; red line indicates the SCIU5 group, $n=8$; blue line indicates the SCIU40 group, $n=7$ ). (b) Fine motor coordination was evaluated using the ladder rung test based on the percentage of errors, defined as a slip, miss, or drag. (c, d) Transverse spinal cord sections obtained 8 weeks after $\mathrm{SCl}$ were stained with $\mathrm{H} \& \mathrm{E}$ to evaluate the area of tissue injury and cavity. The injured cavity area is marked with a dashed red line, and the red box in C is magnified 20x in D. (e) A total of five slices from each of three rats were selected, and the cavity area following contusion injury was quantified. (f) Tissue density was assessed using H\&E-stained slices based on four regions of interest per spinal cord tissue in five slices from each of three rats, excluding the necrotic zone. (g) FJC staining of lesion areas in transverse sections of the spinal cord and the enlarged images at high magnification $(\times 200$, red square box). (h, i) FJC staining was evaluated quantitatively based on the number of FJC/DAPI co-labeled cells and fluorescence intensity in six regions of interest per spinal cord tissue in five slices from each of three rats. Data are expressed as means \pm SEM. Two-way ANOVA and Turkey's tests for the multiple comparisons were used. ${ }^{*} \mathrm{P}<0.05,{ }^{\star} * \star \mathrm{P}<0.001,{ }^{\star \star \star *} \mathrm{P}<0.0001$ compared with the sham control, \#P< $0.05, \# \# P<0.01, \# \# \#$ P $<0.0001$ compared with the SCIU5-treated group.

\section{Supplementary Files}

This is a list of supplementary files associated with this preprint. Click to download.

- Supplementaryfigures.pdf 